TRANSACTIONS OF THE

AMERICAN MATHEMATICAL SOCIETY

Volume 357, Number 7 , Pages 2909-2938

S 0002-9947(04)03769-9

Article electronically published on December 29, 2004

\title{
NONLINEAR SCHRÖDINGER EQUATIONS WITH HARDY POTENTIAL AND CRITICAL NONLINEARITIES
}

\author{
DIDIER SMETS
}

\begin{abstract}
We study a time-independent nonlinear Schrödinger equation with an attractive inverse square potential and a nonautonomous nonlinearity whose power is the critical Sobolev exponent. The problem shares a strong resemblance with the prescribed scalar curvature problem on the standard sphere. Particular attention is paid to the blow-up possibilities, i.e. the critical points at infinity of the corresponding variational problem. Due to the strong singularity in the potential, some new phenomenon appear. A complete existence result is obtained in dimension 4 using a detailed analysis of the gradient flow lines.
\end{abstract}

\section{INTRODUCTION}

We will consider the following nonlinear Schrödinger equation:

$$
\left\{\begin{aligned}
-\Delta u-\frac{\lambda}{|x|^{2}} u & =K(x) u^{2^{*}-1}, \\
u>0, & u \in \mathcal{D}^{1,2}\left(\mathbb{R}^{N}\right),
\end{aligned}\right.
$$

where $N \geq 3,2^{*}:=2 N /(N-2)$ is the classical Sobolev exponent, and the energy space $\mathcal{D}^{1,2}\left(\mathbb{R}^{N}\right):=\left\{u \in L_{\text {loc }}^{2}\left(\mathbb{R}^{N}\right)\right.$ s.t. $\left.\nabla u \in L^{2}\left(\mathbb{R}^{N}\right)\right\}$. The parameter $\lambda$ is positive and $K$ will be at least positive, bounded and bounded away from zero.

The main interest of this equation, in addition to the critical Sobolev exponent, is the presence of the so-called "inverse square potential" in the linear part. Similar critical nonlinear Schrödinger equations, but with bounded, periodic, coercive or $L^{N / 2}$ potentials, have been studied by many authors, starting with the pioneering work of P.-L. Lions 22. In our case, the noncompactness of the embedding $\mathcal{D}^{1,2}\left(\mathbb{R}^{N}\right) \rightarrow L^{2}\left(|x|^{-2} d x\right)$, even locally in any neighborhood of zero, leads to other additional difficulties, and, more importantly, to new phenomenon concerning the possibility of blow-up.

Noncompact variational problems have attracted much attention since the late seventies. Among them, the Yamabe [29] and the prescribed scalar curvature problems have played an important role. For those, but also for many related elliptic equations, the loss of compactness is caused by the invariant action of the conformal group, or of one of its subgroups, leading to possible spikes formation.

To overcome this difficulty, the a priori knowledge of the energy range where the Palais-Smale condition holds is helpful, and sometimes suffices to construct

Received by the editors February 11, 2002 and, in revised form, January 13, 2004.

2000 Mathematics Subject Classification. Primary 35J10, 35J70, 58J37.

Key words and phrases. Hardy potential, critical Sobolev exponent, prescribed scalar curvature. 
critical points. The Yamabe equation falls into this category, and was solved in complementing cases by T. Aubin [1] and R. Schoen [24]. They proved that the minimum of the corresponding constrained variational problem always stands below the first critical level for the Palais-Smale condition, unless the manifold is the the standard sphere in which case the equation has a trivial solution. Instead, for the prescribed scalar curvature problem on the standard sphere, the Palais-Smale condition does not hold at any level which could lead to a critical point, and a further analysis has to be done; the picture is much less complete. In this direction, one can say that basically two techniques are under hand.

The first one, due essentially to A. Bahri (see e.g. 3]), consists of a careful analysis of the gradient flow lines in the neighborhood of the so-called "critical points at infinity". It has lead to beautiful results, like the one by A. Bahri and J.-M. Coron [5], where it was used together with topological arguments.

A second one, initiated by R. Schoen [25], is the fine analysis of blow-up, where true critical points of approximating problems are considered instead of general Palais-Smale sequences. This leads to easier estimates as well as Pohozaev-type identities. Later important developments of this method include works of Y.Y. Li [19, 20], E. Hebey and M. Vaugon [17, Druet [13, 14, 15, and Z.C. Han [16].

Equation (11), after identification of $\mathbb{R}^{N}$ with the standard $N$-dimensional sphere through the conformal stereographic projection, can be considered as a variant of the prescribed scalar curvature equation on $S^{N}$

$$
-\Delta_{g} u+R(x) u=K(x) u^{2^{*}-1}
$$

where $R$ is no longer the initial constant curvature but a function with two attractive singularities located at the north and south pole. At the limit $\lambda=0$, we find the usual prescribed scalar curvature equation. Equations like (2) were considered by A. Bahri and H. Brezis [4] and also by Y.Y. Li and M. Zhu 21] in the case of a bounded function $R(x)$.

The simplest and governing case for (1) is the one with $K \equiv 1$. It has been studied by S. Terracini [27], where it was shown in particular that an explicit solution exists for all $0<\lambda<(N-2)^{2} / 4$, namely

$$
U_{\lambda}(x):=\frac{c_{\lambda}}{\left(|x|^{1-a}\left(1+|x|^{2 a}\right)\right)^{\frac{N-2}{2}}}
$$

where $a:=\sqrt{1-4 \lambda /(N-2)^{2}}$ and $c_{\lambda}$ is an appropriate constant. This solution is also known to be the unique positive solution, up to a conformal transformation of the form $U_{\lambda}^{\varepsilon}:=\varepsilon^{(2-N) / 2} U_{\lambda}(x / \varepsilon)$, for some $\varepsilon \in \mathbb{R}_{*}^{+}$.

It is worth noting that $U_{\lambda}$ does not belong to $L^{\infty}\left(\mathbb{R}^{N}\right)$ as soon as $\lambda>0$, because of the singularity in zero. For this reason, it seems difficult to handle (1) in the general case using the "fine analysis of blow-up" technique quoted here above. Indeed, this technique essentially relies on obtaining uniform $L^{\infty}$ a priori bounds on solutions of approximating problems, after having ruled out any blow-up possibility; this is of course hopeless here.

After fixing some useful notations, Section 2 starts with some nonexistence results which help in setting the hypotheses on $\lambda$ and $K$. Regularity properties for weak solutions of (11) are also established, following an extension for Hardy potentials of the theorem of Brezis and Kato 9]. For future use, we also recall at the end of the section some known properties of (1) with $K \equiv 1$ and $\lambda=0$ or $0<\lambda<(N-2)^{2} / 4$. 
Section 3 is devoted to the analysis of the noncompactness. Roughly speaking, we prove that blowing up positive Palais-Smale sequences can bear exactly two kind of bubbles. Up to harmless constants, these are either of the form

or

$$
U_{0}^{\varepsilon_{n}, y_{n}}(x):=\varepsilon_{n}^{\frac{2-N}{2}} U_{0}\left(\frac{x-y_{n}}{\varepsilon_{n}}\right) \quad \text { with } \frac{\left|y_{n}\right|}{\varepsilon_{n}} \rightarrow+\infty
$$

$$
U_{\lambda}^{\varepsilon_{n}, y_{n}}(x):=\varepsilon_{n}^{\frac{2-N}{2}} U_{\lambda}\left(\frac{x-y_{n}}{\varepsilon_{n}}\right) \quad \text { with } \frac{\left|y_{n}\right|}{\varepsilon_{n}} \rightarrow 0 .
$$

The first kind, which we will call "classical blow-up", corresponds to the usual instanton studied in Yamabe-type equations. The second one, which we will call singularity blow-up, is particular to this problem, and is originated in the Hardy potential. An important step of the analysis is to exclude a priori the possibility of an intermediate-type blow-up, which looks like

$$
V_{n}(x):=\varepsilon_{n}^{(2-N) / 2} V\left(\left(x-y_{n}\right) / \varepsilon_{n}\right)
$$

for some profile $V$ and scaling sequences satisfying $\left|y_{n}\right| / \varepsilon_{n} \rightarrow c$ for some $0<c<$ $+\infty$. The analysis being complete, we obtain the detailed picture of the energy levels where positive Palais-Smale sequences may fail to give rise to solutions.

Using the characterization of Section 3, we obtain existence results in Section 4, when Palais-Smale sequences can be constructed at safe energy levels. Basically, this happens in the general case when $\lambda$ is close to either zero or $(N-2)^{2} / 4$. It also happens when $K$ is radial, in which case the classical blow-up can be easily ruled out, or when $K$ satisfies some sign condition with respect to its limits at zero and infinity, in which case the problem is easily solved by constrained minimization.

Section 5 is devoted to the remaining case, for intermediate values of $\lambda$. In this situation, the energy level of the Palais-Smale sequence that we construct may fall just inside the region where a classical blow-up is not excluded. Most of the section consists of a careful analysis of the gradient flow lines in the neighborhood of the critical points at infinity, a method largely developed by A. Bahri. Writing a function in such a neighborhood under the classical form

$$
u=c\left(U_{0}^{\varepsilon, y}+\delta v\right), \quad \text { with } \quad\left\langle U_{0}^{\varepsilon, y}, v\right\rangle=\left\langle\partial_{\varepsilon} U_{0}^{\varepsilon, y}, v\right\rangle=\left\langle\partial_{x_{i}} U_{0}^{\varepsilon, y}, v\right\rangle=0
$$

and $\|v\|=1$, the key step here is to estimate optimally the interaction terms of the form

$$
\int_{\mathbb{R}^{N}} \frac{U_{0}^{\varepsilon, y} v}{|x|^{2}} \quad \text { or } \quad \int_{\mathbb{R}^{N}} \frac{\partial_{\varepsilon} U_{0}^{\varepsilon, y} v}{|x|^{2}} .
$$

Up to now, dimension four is the only one for which we can completely achieve all the analysis: some of the intermediate results will thus also be stated only in that case. Denoting by $\sigma$ the stereographic projection from $S^{4}$ to $\mathbb{R}^{4} \cup \infty$, our main theorem reads:

Theorem. Assume that $N=4$ and that $K \circ \sigma \in \mathcal{C}^{2}\left(S^{4}\right)$ is positive and such that $K(0)=\lim _{|x| \rightarrow+\infty} K(x)$. Then (1) has at least one positive solution $u \in \mathcal{D}^{1,2}\left(\mathbb{R}^{N}\right)$ for all $0<\lambda<1$, and no positive solution if $\lambda \geq 1$.

In dimension 3 , the above-mentioned interaction term cannot be better estimated than what follows from the Cauchy-Schwarz inequality, leading to additional difficulties. From dimension 5, we suspect a similar theorem to hold, under some

\footnotetext{
${ }^{1}$ Added in proof: recently the author together with $\mathrm{K}$. Sandeep completed the case $N=3$.
} 
nondegeneracy conditions at the critical points of $K$. These remaining cases will be the object of a further study, as well as the case $\lambda<0$.

\section{Hypotheses AND NOTATIONS}

Notations. In this paper, the ball of center $x$ and radius $r$ is denoted by $B(x, r)$, or simply $B(r)$ if no confusion follows. Various positive constants whose exact values are meaningless are denoted by $C$. The functional space we use is

$$
\mathcal{D}^{1,2}\left(\mathbb{R}^{N}\right):=\left\{u \in L_{\text {loc }}^{2}\left(\mathbb{R}^{N}\right) \text { such that } \nabla u \in L^{2}\left(\mathbb{R}^{N}\right)\right\}
$$

which, endowed with the scalar product $\langle u, v\rangle:=\int_{\mathbb{R}^{N}} \nabla u \nabla v$, is a Hilbert space. The pairing between $\mathcal{D}^{1,2}\left(\mathbb{R}^{N}\right)$ and its dual is also denoted by $\langle\cdot, \cdot\rangle$. The norm in $\mathcal{D}^{1,2}\left(\mathbb{R}^{N}\right)$ is written $\|\cdot\|$ while the $L^{p}$ norms are written $\|\cdot\|_{p}$. The space $\mathcal{D}(\Omega)$ is the one of all infinitely differentiable functions having compact support in $\Omega$. An expression depending on $\varepsilon>0$ will be said to be $O(\varepsilon)$ (resp. $o(\varepsilon)$ ) if, all other parameters being fixed, its quotient by $\varepsilon$ is bounded in absolute value by a positive constant (resp. tends to 0 ) when $\varepsilon \rightarrow 0$. It will be said to be $O_{+}(\varepsilon)$ if it is positive and bounded above and below by two positive constants times $\varepsilon$ when $\varepsilon \rightarrow 0$.

We start by fixing the assumptions on the two parameters in (1), namely $\lambda$ and $K$. The behavior of (1) is much affected by the sign of $\lambda$. Indeed, if we write the linear part as $-\Delta u+V(x) u$, it acts on the potential term $V(x)$ making it attractive when $\lambda>0$ and repulsive when $\lambda<0$. We will focus on the attractive case $\lambda>0$, which, on an analytical point of view, seems to be the richest one. We will also always assume that $K$ is at least positive and twice differentiable. This is not essential in the whole part of the analysis, but we want to avoid lengthy assumptions and (or) numerous particular results.

Concerning $\lambda$, the following first nonexistence result holds. It is probably already mentioned in the literature, but we have it here for the sake of completeness and because of the simplicity of the proof.

Proposition 2.1. Assume $\lambda \geq(N-2)^{2} / 4$. Then there is no positive solution of the inequation

$$
-\Delta u-\lambda \frac{u}{|x|^{2}} \geq 0
$$

which is in $H_{\mathrm{loc}}^{1}(\Omega)$ for some neighborhood $\Omega$ of zero.

Proof. Define $v$ on $\mathbb{R} \times S^{n-1}$ by

$$
u(x)=|x|^{\frac{2-N}{2}} v\left(\frac{N-2}{2} \log r, \theta\right),
$$

where as usual $(r, \theta)$ are radial and spherical coordinates for $x$. Notice that since $u$ is defined in some neighborhood of zero, $v$ is at least defined on $(-\infty, a) \times S^{N-1}$ for some $a \in \mathbb{R}$.

A straightforward computation shows that the inequation for $u$ multiplied by $|x|^{(N+2) / 2}$ yields, in terms of $v$,

$$
-\Delta_{g} v \geq\left(\lambda-(N-2)^{2} / 4\right) v \geq 0 .
$$

Here $\Delta_{g}$ denotes the Laplace-Beltrami operator on $\mathbb{R} \times S^{N-1}$. Let

$$
w(s):=\int_{S^{N-1}} v(s, \theta) d \theta .
$$


Then

$$
\begin{aligned}
-w^{\prime \prime}(s) & =\int_{S^{N-1}}-\frac{\partial^{2} v}{\partial r^{2}}(s, \theta) d \theta \\
& =\int_{S^{N-1}}-\Delta_{g} v(s, \theta) d \theta+\int_{S^{N-1}}-\Delta_{\theta} v(s, \theta) d \theta \\
& =\int_{S^{N-1}}-\Delta_{g} v(s, \theta) d \theta \geq 0 .
\end{aligned}
$$

Since $-w^{\prime \prime} \geq 0$ and $w$ is positive, we infer that $\lim _{s \rightarrow-\infty} w(s)=c>0$. This implies that

$$
\int_{S^{N-1}} u(r, \theta) d \theta \geq c r^{(2-N) / 2}
$$

for $r$ sufficiently small, so that

$$
\begin{aligned}
\int_{B(0, r)} u^{2^{*}}(x) d x & \geq C \int_{0}^{r}\left(\int_{S^{N-1}} u(r, \theta) d \theta\right)^{2^{*}} r^{N-1} d r \\
& \geq C \int_{0}^{r} r^{-1} d r=+\infty .
\end{aligned}
$$

This of course is impossible if $u \in H^{1}(B(0, r))$.

Concerning $K$, the following Pohozaev type identity holds:

Proposition 2.2. Assume $K$ is continuously differentiable and let $u \in \mathcal{D}^{1,2}\left(\mathbb{R}^{N}\right)$ be a solution of (11). Then

$$
\int_{\mathbb{R}^{N}}\langle\nabla K(x), x\rangle u^{2^{*}} d x=0 .
$$

In particular, a necessary condition for the existence of a positive solution is that $\partial K / \partial r$ changes sign at least at one point.

Proof. The proof is classical in the case $\lambda=0$, and due to its invariance under the scaling $u \mapsto \varepsilon^{(2-N) / 2} u(x / \varepsilon)$, the Hardy term does not add any contribution. The only difference with respect to the classical proof is the lack of regularity of the solution. Indeed, it is generally assumed that $u$ belongs to $H_{\text {loc }}^{2}$, whereas we just have this regularity out of the origin here, due to the Hardy term. To overcome this difficulty, one can multiply $u$ by a cut-off function $\psi_{n} \in \mathcal{D}\left(\mathbb{R}^{N} \backslash\{0\}\right)$ such that $\psi_{n} \equiv 1$ on $B(n) \backslash B(1 / n), \psi_{n} \equiv 0$ on $B(1 /(2 n)) \cup \mathbb{R}^{N} \backslash B(2 n)$, and $\left|x \cdot \nabla \psi_{n}\right| \leq C$ everywhere. It is then easy to check that all the estimates in [28], Theorem B.3, pass to the limit.

The preceding necessary condition is probably too weak to be used in practice. Instead, we will assume that $K$ has a limit when $|x| \rightarrow \infty$ (called $K(\infty)$ ) which is equal to $K(0)$. Clearly this implies that $\partial K / \partial r$ changes sign, unless $K$ is constant. Without loss of generality, we can also assume the normalizing condition $K(0)=1$, the general case being reduced to this one by a scaling.

Summarizing, the following hypotheses will be assumed to hold throughout the paper, where $\sigma$ refers to the stereographic projection:

$$
\left\{\begin{array}{l}
0<\lambda<(N-2)^{2} / 4 \\
K \circ \sigma \in \mathcal{C}^{2}\left(S^{N}\right) \text { is positive, } \\
K(0)=\lim _{|x| \rightarrow+\infty} K(x)=1 .
\end{array}\right.
$$


Given these hypotheses, we now define the $\mathcal{C}^{2}$ functional

$$
J: \mathcal{D}^{1,2}\left(\mathbb{R}^{N}\right) \rightarrow \mathbb{R}, u \mapsto \int_{\mathbb{R}^{N}} \frac{|\nabla u|^{2}}{2}-\frac{\lambda}{2} \frac{u^{2}}{|x|^{2}}-K(x) \frac{|u|^{2^{*}}}{2^{*}} d x,
$$

whose critical points are weak solutions of (1), without the sign condition. Concerning the regularity of weak solutions, the following extension of the Brezis-Kato theorem [9] can be proved:

Theorem 2.3. Let $\Omega$ be a bounded neighborhood of zero in $\mathbb{R}^{N}, 0<\lambda<(N-2)^{2} / 4$, $V \in L^{N / 2}(\Omega)$ and $g \in L^{q}(\Omega), q \geq 2$. If $u \in H_{0}^{1}(\Omega)$ is a weak solution of

$$
-\Delta u-\lambda \frac{u}{|x|^{2}}-V(x) u+\nu u=g \text { in } \Omega,
$$

where $\nu$ is such that the linear operator on the left-hand side is positive, then

$$
u \in \bigcap_{p<p_{\lim }} L^{p}(\Omega), \quad \text { with } \quad p_{\lim }=2^{*} \min \left(q / 2, \frac{1}{1-\sqrt{1-4 \lambda /(N-2)^{2}}}\right) .
$$

Proof. First notice that since $\lambda<(N-2)^{2} / 4$ and $V \in L^{N / 2}$, the operator $-\Delta-$ $\left(\lambda /|x|^{2}+V\right) I$ is semibounded so that for large $\nu$ the operator in the equation is positive. We can also assume that $g$ is positive, the general case being obtained by decomposing $g:=g^{+}-g^{-}$.

We proceed as in [9]. Let $W_{k}:=\min \left\{k, \lambda /|x|^{2}+V(x)\right\}$ and let $u_{k}$ be the unique solution of

$$
\left\{\begin{array}{l}
-\Delta u_{k}-W_{k}(x) u_{k}+\nu u_{k}=g \text { in } \Omega \\
\\
u_{k} \in H_{0}^{1}(\Omega)
\end{array}\right.
$$

Clearly, $u_{k}$ is positive. Set $u_{k}^{n}:=\min \left(n, u_{k}\right)$ and assume that $u_{k} \in L^{p}(\Omega)$ for some $p \leq q$. Notice that $\left(u_{k}^{n}\right)^{p-1} \in H_{0}^{1}(\Omega)$ so that multiplying ([6) by $\left(u_{k}^{n}\right)^{p-1}$ in the weak sense we obtain

$$
\begin{aligned}
(p-1) \int_{\Omega}\left(u_{k}^{n}\right)^{p-2}\left|\nabla u_{k}^{n}\right|^{2} d x \leq \int_{\Omega} W_{k}^{+} & (x)\left(u_{k}^{n}\right)^{p} d x \\
& \quad+\int_{u_{k}>n} k n^{p-1} u_{k} d x+\int_{\Omega} g\left(u_{k}^{n}\right)^{p-1} d x .
\end{aligned}
$$

Using the Hardy inequality and the fact that for each $\varepsilon>0$ there exists $C_{\varepsilon}>0$ such that

$$
\int_{\Omega}|V(x)| v^{2} d x \leq \varepsilon\|\nabla v\|_{2}^{2}+C_{\varepsilon}\|v\|_{2}^{2},
$$

inequality (7) can be rewritten as

(8)

$$
\begin{aligned}
\frac{4(p-1)}{p^{2}}\left\|\nabla\left(\left(u_{k}^{n}\right)^{p / 2}\right)\right\|_{2}^{2} \leq & C\|g\|_{q}\left\|u_{k}^{n}\right\|_{p}^{p-1}+\left(4 \lambda /(N-2)^{2}+\varepsilon\right)\left\|\nabla\left(\left(u_{k}^{n}\right)^{p / 2}\right)\right\|_{2}^{2} \\
& +C_{\varepsilon}\left\|u_{k}^{n}\right\|_{p}^{p}+k \int_{u_{k}>n}\left(u_{k}\right)^{p} d x
\end{aligned}
$$

If $p<2 p_{\lim } / 2^{*}$, it is easy to compute that

$$
4 \lambda /(N-2)^{2}<\frac{4(p-1)}{p^{2}},
$$


hence, choosing $\varepsilon$ sufficiently small and using the Sobolev inequality, we infer that

$$
\left\|u_{k}^{n}\right\|_{2^{*} p / 2}^{p} \leq C_{p}\left[\|g\|_{q}+\left\|u_{k}^{n}\right\|_{p}^{p}+\int_{u_{k}>n}\left(u_{k}\right)^{p} d x\right] .
$$

Since by assumption $u_{k} \in L^{p}$, taking the limit as $n \rightarrow+\infty$ yields

$$
\left\|u_{k}\right\|_{2^{*} p / 2}^{p} \leq C_{p}\left[\|g\|_{q}+\left\|u_{k}\right\|_{p}^{p}\right.
$$

so that $u_{k} \in L^{2^{*} p / 2}(\Omega)$. The above estimates being uniform with respect to $k$, we deduce that $u \in L^{2^{*} p / 2}$.

Starting with $p=2$, we can recursively use the above process to improve the integrability order of $u$, as long as $p<2 p_{\lim } / 2^{*}$. The result follows.

Corollary 2.4. Any solution $u$ of (1) satisfies

$$
u \in \bigcap_{p<p_{\lim }} L_{\mathrm{loc}}^{p}\left(\mathbb{R}^{N}\right), \quad \text { with } \quad p_{\lim }=2^{*} \frac{1}{1-\sqrt{1-4 \lambda /(N-2)^{2}}} .
$$

Proof. Let $R>0$ and let $\psi \in \mathcal{D}(B(R))$ be a cut-off function such that $\psi \equiv 1$ on $B(R / 2)$ and $\|\psi\|_{\infty}=1$. Then, $w:=u \psi$ satisfies the following equation in the weak sense:

$$
-\Delta w-\lambda \frac{w}{|x|^{2}}-V(x) w+\nu w=g, \quad w \in H_{0}^{1}(B(R)),
$$

where

$$
V(x):=K(x) u^{2^{*}-2} \in L^{N / 2}(B(R)) \quad \text { and } \quad g:=-2 \nabla u \nabla \psi-u \Delta \psi-\nu w .
$$

Using the Brezis-Kato theorem and elliptic regularity theory, we infer that $u \in$ $W_{\text {loc }}^{2, p}(B(R) \backslash\{0\})$ for any $p<\infty$, and hence that $w \in \mathcal{C}^{1}(B(R) \backslash\{0\})$. Since $\nabla \psi \equiv 0$ on $B(R / 2),-2 \nabla u \nabla \psi-u \Delta \psi \in \mathcal{C}^{1}(B(R))$ and the $L^{p}$ regularity of $g$ is given by that of $w$. Starting from $g \in L^{2}$ and arguing recursively, we improve the regularity of $w$ using Theorem 2.3 hence the one of $g$ by what precedes, and the argument goes on until we reach the limitation imposed by $\lambda$. The proof is complete.

Due to the homogeneity in the nonlinearity, we can also associate with the functional $J$ a "natural constraint", often called the Nehari manifold:

$$
\mathcal{N}:=\left\{u \in \mathcal{D}^{1,2}\left(\mathbb{R}^{N}\right) \text { such that }\left\langle J^{\prime}(u), u\right\rangle=0\right\},
$$

which turns out to be a $\mathcal{C}^{2}$ sub-manifold of $\mathcal{D}^{1,2}\left(\mathbb{R}^{N}\right)$. The set of positive functions in $\mathcal{N}$ is called $\mathcal{N}^{+}$. It is easily seen that $\mathcal{N}$ is symmetric with respect to the origin and has exactly one intersection (up to reflection) with each one-dimensional subspace of $\mathcal{D}^{1,2}\left(\mathbb{R}^{N}\right)$. Calling $u$ this intersection point, we have the characterization

$$
J(u)=\max _{t \in \mathbb{R}^{+}} J(t u) .
$$

On $\mathcal{N}, J$ can be rewritten under the form

$$
J(u)=\left(\frac{1}{2}-\frac{1}{2^{*}}\right)\left(\int_{\mathbb{R}^{N}}|\nabla u|^{2}-\lambda \frac{u^{2}}{|x|^{2}} d x\right)^{N / 2}\left(\int_{\mathbb{R}^{N}} K(x)|u|^{2^{*}} d x\right)^{(2-N) / 2},
$$

which has the advantage of being homogeneous of order zero. It is then sometimes useful to consider the extension of this homogeneous functional to the whole $\mathcal{D}^{1,2}\left(\mathbb{R}^{N}\right)$; we call it $\tilde{J}$.

The following trivial proposition will be helpful to ensure the positivity of solutions constructed through minimax theorems. 
Proposition 2.5. There is a continuous mapping $P: \mathcal{N} \rightarrow \mathcal{N}^{+}$such that $P(u)=u$ if $u$ is positive and $J(P(u)) \leq J(u)$ for all $u \in \mathcal{N}$.

Proof. Define $P(u)$ as the unique multiple of $|u|$ which belongs to $\mathcal{N}$. It is easily seen that $P$ is continuous and

$$
J(P(u))=\tilde{J}(|u|) \leq \tilde{J}(u)=J(u)
$$

for each $u \in \mathcal{N}$.

Before going into the study of Palais-Smale sequences for $J$, we need to introduce some limiting problems which play an important role in this analysis, and recall some of their properties. The first one is the Yamabe equation on $\mathbb{R}^{N}$,

$$
-\Delta u=u^{2^{*}-1}, \quad u \in \mathcal{D}^{1,2}\left(\mathbb{R}^{N}\right),
$$

whose set of positive solutions is the well-known $(N+1)$-parameters family of instantons

where

$$
U_{0}^{\varepsilon, y}(x):=\varepsilon^{(2-N) / 2} U_{0}\left(\frac{x-y}{\varepsilon}\right),
$$

$$
U_{0}(x):=c(N)\left(1+|x|^{2}\right)^{\frac{2-N}{2}}
$$

for some appropriate constant $c(N)>0$. These solutions are also known to minimize the Sobolev quotient

$$
S_{0}:=\min _{u \in \mathcal{D}^{1,2}\left(\mathbb{R}^{N}\right) \backslash\{0\}} \frac{\int_{\mathbb{R}^{N}}|\nabla u|^{2} d x}{\left(\int_{\mathbb{R}^{N}}|u|^{2^{*}} d x\right)^{2 / 2^{*}}},
$$

whose exact value was determined by Aubin [2].

The second limiting equation is

$$
-\Delta u-\lambda \frac{u}{|x|^{2}}=u^{2^{*}-1}, \quad u \in \mathcal{D}^{1,2}\left(\mathbb{R}^{N}\right) .
$$

For $0<\lambda<(N-2)^{2} / 4$, it has been studied by Terracini [27, where it is shown that all the positive solutions are of the form $U_{\lambda}^{\varepsilon}(x):=\varepsilon^{(2-N) / 2} U_{\lambda}(x / \varepsilon)$, where

$$
U_{\lambda}(x):=c_{\lambda}(N)\left(\frac{|x|^{a-1}}{1+|x|^{2 a}}\right)^{\frac{N-2}{2}}
$$

for an appropriate constant $c_{\lambda}(N)>0$, and $a:=\sqrt{1-4 \lambda /(N-2)^{2}}$. Notice that this time the translation parameter has disappeared, the equation being no longer autonomous. Notice also that $U_{\lambda}$ has an unbounded singularity at the origin. These solutions are also minimizers for the quotients

$$
S_{\lambda}:=\min _{u \in \mathcal{D}^{1,2}\left(\mathbb{R}^{N}\right) \backslash\{0\}} \frac{\int_{\mathbb{R}^{N}}\left[|\nabla u|^{2}-\lambda \frac{u^{2}}{|x|^{2}}\right] d x}{\left(\int_{\mathbb{R}^{N}}|u|^{2^{*}} d x\right)^{2 / 2^{*}}} .
$$

Clearly, $S_{\lambda}$ is decreasing in $\lambda$, and a simple computation shows that

$$
\lim _{\lambda \rightarrow(N-2)^{2} / 4} S_{\lambda}=0
$$

For convenience, we also define the following quantities, which will represent the amount of $J$ carried over by blowing-up bubbles:

$$
D_{0}:=\int_{\mathbb{R}^{N}}\left[\frac{1}{2}\left|\nabla U_{0}\right|^{2}-\frac{1}{2^{*}} U_{0}^{2^{*}}\right] d x=\frac{1}{2} S_{0}^{N / 2}
$$


and

$$
D_{\lambda}:=\int_{\mathbb{R}^{N}}\left[\frac{1}{2}\left(\left|\nabla U_{\lambda}\right|^{2}-\lambda \frac{U_{\lambda}^{2}}{|x|^{2}}\right)-\frac{1}{2^{*}} U_{\lambda}^{2^{*}}\right] d x=\frac{1}{2} S_{\lambda}^{N / 2} .
$$

With these precisions, we are now ready for the next section where we analyze the possible loss of compactness in (1).

\section{NONCOMPACTNESS ANALYSIS}

Characterizations of Palais-Smale sequences for equations related to (10) were obtained after the works of Sacks-Uhlenbeck [23], Struwe [26] and others. For those equations, the noncompactness is completely described by the single blow-up profile $U_{0}$. This possibility is still present for (1); indeed it is easily verified that any sequence of the form

$$
u_{n}(x):=K(y)^{\frac{2-N}{4}} \varepsilon_{n}^{\frac{2-N}{2}} U_{0}\left(\frac{x-y_{n}}{\varepsilon_{n}}\right)
$$

is a Palais-Smale sequence for $J$ if $y_{n} \rightarrow y \neq 0$ and $\varepsilon_{n} \rightarrow 0$. If $y=0$, a further computation easily shows that $\left(u_{n}\right)$ remains a Palais-Smale sequence if in addition $\varepsilon_{n} /\left|y_{n}\right| \rightarrow 0$.

New to (11) is the second blow-up possibility. Indeed, any sequence of the form

$$
u_{n}(x):=\varepsilon_{n}^{\frac{2-N}{2}} U_{\lambda}\left(\frac{x-y_{n}}{\varepsilon_{n}}\right)
$$

is a Palais-Smale sequence for $J$ if $y_{n} \rightarrow 0$ and $\varepsilon_{n} /\left|y_{n}\right| \rightarrow+\infty$.

In the first kind of blow-up, the term in $\frac{u_{n}^{2}}{x^{2}}$ completely disappears at the limit, while in the second kind it remains completely unaffected. A natural question is the possibility of an intermediate blow-up, with an appropriate profile, where $\varepsilon_{n} /\left|y_{n}\right|$ would be asymptotically constant and nonzero.

The next theorem excludes this last possibility, and completely characterizes positive Palais-Smale sequences for $J$. Its proof follows a series of lemmas which are collected in the Appendix.

Theorem 3.1. Let $\left(u_{n}\right) \subset \mathcal{D}^{1,2}\left(\mathbb{R}^{N}\right), u_{n} \geq 0$, be a Palais-Smale sequence for $J$ at level $d \geq 0$. Then, there are $k, l \in \mathbb{N}, l$ sequences $\left(R_{n}^{j}\right) \subset \mathbb{R}_{*}^{+}(1 \leq j \leq l), 2 k$ sequences $\left(r_{n}^{j}\right) \subset \mathbb{R}_{*}^{+}$and $\left(y_{n}^{j}\right) \subset \mathbb{R}^{N} \backslash\{0\}(1 \leq j \leq k)$, and $0 \leq u \in \mathcal{D}^{1,2}\left(\mathbb{R}^{N}\right)$ such that, up to a subsequence:

- $J^{\prime}(u)=0$,

- $y_{n}^{j} \rightarrow y^{j} \in \mathbb{R}^{N} \cup\{\infty\} \quad$ and $\frac{r_{n}^{j}}{\left|y_{n}^{j}\right|} \rightarrow 0 \quad(1 \leq j \leq k)$,

- $R_{n}^{j} \rightarrow 0$ or $R_{n}^{j} \rightarrow+\infty \quad(1 \leq j \leq l)$,

- $d=J(u)+\sum_{j=1}^{k} K\left(y^{j}\right)^{\frac{2-N}{2}} D_{0}+l D_{\lambda}$,

- $\left\|u_{n}-u-\sum_{j=1}^{k} K\left(y^{j}\right)^{\frac{2-N}{4}} U_{0}^{r_{n}^{j}, y_{n}^{j}}-\sum_{j=1}^{l} U_{\lambda}^{R_{n}^{j}, 0}\right\| \rightarrow 0$.

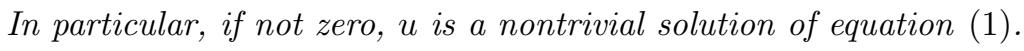

Remark 3.2. A similar theorem can be proved without the sign condition for $\left(u_{n}\right)$, but it would be of no additional use here since the classification of all the sign changing solutions of the limiting equations is not available. 
Corollary 3.3. Any positive Palais-Smale sequence for $J$ at a level which is not of the form

$$
\sum_{j=1}^{k} K\left(y^{j}\right)^{\frac{2-N}{2}} D_{0}+l D_{\lambda}
$$

for arbitrary naturals $k, l$ and points $y^{j} \in \mathbb{R}^{N}$, gives rise to a nontrivial weak solution of equation (1).

Proof of Theorem 3.1. 1) We have

$$
\begin{aligned}
d+1+o\left(\left\|u_{n}\right\|\right) & =J\left(u_{n}\right)-\frac{1}{2^{*}}\left\langle J^{\prime}\left(u_{n}\right), u_{n}\right\rangle \\
& =\left(\frac{1}{2}-\frac{1}{2^{*}}\right) \int_{\mathbb{R}^{N}}\left[\left|\nabla u_{n}\right|^{2}-\lambda \frac{u_{n}}{|x|^{2}}\right] d x \geq c(\lambda)\left\|u_{n}\right\|^{2},
\end{aligned}
$$

so that $\left(u_{n}\right)$ is bounded in $\mathcal{D}^{1,2}\left(\mathbb{R}^{N}\right)$. Up to a subsequence, we can assume that there exists $u \in \mathcal{D}^{1,2}\left(\mathbb{R}^{N}\right)$ such that $u_{n} \rightarrow u$ in $\mathcal{D}^{1,2}\left(\mathbb{R}^{N}\right)$ and $u_{n}(x) \rightarrow u(x)$ almost everywhere.

2) We deduce from Lemma A.2 that $J^{\prime}(u)=0$ and that $v_{n}:=u_{n}-u$ is a Palais-Smale sequence for $J$ at level $d-J(u)$. Notice that $v_{n} \rightarrow 0$.

3) We first treat the "tight" case where $\left(v_{n}\right) \subset H_{0}^{1}(B(R))$ for some fixed ball of radius $R>0$. The remaining case will be obtained by a splitting argument together with a Kelvin transform.

If $v_{n} \rightarrow 0$ in $L^{2^{*}}\left(\mathbb{R}^{N}\right)$, we obtain

$$
\left\|v_{n}\right\|^{2} \leq C \int_{\mathbb{R}^{N}}\left[\left|\nabla v_{n}\right|^{2}-\lambda \frac{v_{n}}{|x|^{2}}\right] d x=C \int_{\mathbb{R}^{N}} K(x)\left|v_{n}\right|^{2^{*}} d x+o(1)=o(1),
$$

so that the thesis is satisfied with $k=l=0$ and the Palais-Smale sequence admits a strongly convergent subsequence.

If not, let $0<\delta<S_{0}^{N / 2}\left(1-4 \lambda /(N-2)^{2}\right)^{N / 2}$ such that

$$
\limsup _{n \rightarrow+\infty} \int_{\mathbb{R}^{N}} K(x)\left|v_{n}\right|^{2^{*}} d x>\delta
$$

Up to a subsequence, let $R_{n}>0$ such that $\int_{B\left(R_{n}\right)} K(x) v_{n}{ }^{2^{*}} d x=\delta, R_{n}$ being minimal with this property. Define $w_{n}(x):=R_{n}{ }^{(N-2) / 2} v_{n}\left(R_{n} x\right)$; clearly $\left\|w_{n}\right\|=\left\|v_{n}\right\|$ and $\int_{B(1)} K\left(R_{n} x\right) w_{n}{ }^{2^{*}} d x=\delta$. Up to a subsequence, there exists $w \in \mathcal{D}^{1,2}\left(\mathbb{R}^{N}\right)$ such that $w_{n} \rightarrow w$ in $\mathcal{D}^{1,2}\left(\mathbb{R}^{N}\right)$ and $w_{n}(x) \rightarrow w(x)$ almost everywhere. We distinguish two cases.

a) Assume $w \neq 0$. Since $v_{n} \rightarrow 0$, we have $R_{n} \rightarrow 0$. Then from Lemma A.3 we infer that $w=U_{\lambda}^{\varepsilon}$ for some $\varepsilon>0$, and that $\tilde{v}_{n}:=v_{n}-U_{\lambda}^{R_{n} \varepsilon}$ is a Palais-Smale sequence for $J$ at level $d-J(u)-D_{\lambda}$. 
b) Assume $w=0$. Let $h \in \mathcal{D}(B(1))$; we have

$$
\begin{aligned}
& \int_{\mathbb{R}^{N}}\left|\nabla\left(w_{n} h\right)\right|^{2} d x=\int_{\mathbb{R}^{N}} \nabla w_{n} \nabla\left(h^{2} w_{n}\right) d x+\int_{\mathbb{R}^{N}} w_{n}^{2}|\nabla h|^{2} d x \\
& =\int_{\mathbb{R}^{N}} \nabla w_{n} \nabla\left(h^{2} w_{n}\right) d x+o(1) \quad\left(w_{n} \rightarrow 0 \text { in } L_{\text {loc }}^{2} \text { by the Rellich theorem }\right) \\
& \leq\left\|\nabla J\left(v_{n}\right)\right\|\left\|h^{2} w_{n}\right\|+\int_{\mathbb{R}^{N}} \lambda \frac{h^{2} w_{n}^{2}}{|x|^{2}} d x+\int_{\mathbb{R}^{N}} K\left(R_{n} x\right) w_{n}{ }^{2^{*}} h^{2} d x+o(1) \\
& \left.\leq \frac{4 \lambda}{(N-2)^{2}} \int_{\mathbb{R}^{N}} \mid \nabla w_{n} h\right)\left.\right|^{2}+S_{0}^{-1}\left(\int_{B(1)} K\left(R_{n} x\right) w_{n}{ }^{2^{*}} d x\right)^{\frac{2}{N}} \int_{\mathbb{R}^{N}}\left|\nabla\left(w_{n} h\right)\right|^{2}+o(1) \\
& \leq(1-\tau) \int_{\mathbb{R}^{N}}\left|\nabla\left(w_{n} h\right)\right|^{2}+o(1) \quad \text { for some } \tau>0, \text { by the choice of } \delta .
\end{aligned}
$$

In particular, we deduce from the Sobolev inequality that for each $0<r<1$,

$$
\int_{B(r)} K\left(R_{n} x\right) w_{n}^{2^{*}} d x \rightarrow 0
$$

From the concentration-compactness principle in the tight case (see [22]), it follows that in the sense of measures

$$
K\left(R_{n} x\right) w_{n}{ }^{2^{*}} 1_{|x| \leq 1} \rightarrow \sum_{j} c_{x_{j}} \delta_{x_{j}}
$$

where the points $x_{j} \in \mathbb{R}^{N}$ satisfy $\left|x_{j}\right|=1$. Let $c:=\max \left(c_{x_{j}}\right)$ and define

$$
Q_{n}(r):=\sup _{y \in \mathbb{R}^{N}} \int_{B(y, r)} K\left(R_{n} x\right) w_{n}^{2^{*}} d x
$$

Clearly, $Q_{n}(\infty)>c / 2$, and, for each $r>0$, it follows from (17) that

$$
\liminf _{n \rightarrow+\infty} Q_{n}(r)>c / 2 \text {. }
$$

Hence, there exist sequences $\left(q_{n}\right),\left(s_{n}\right)$ such that $s_{n} \rightarrow 0,\left|q_{n}\right|>1 / 2$, and

$$
\frac{c}{2}=\sup _{q \in \mathbb{R}^{N}} \int_{B\left(q, s_{n}\right)} K\left(R_{n} x\right) w_{n}^{2^{*}} d x=\int_{B\left(q_{n}, s_{n}\right)} K\left(R_{n} x\right) w_{n}{ }^{2^{*}} d x .
$$

Define $z_{n}(x):=s_{n}{ }^{(N-2) / 2} w_{n}\left(s_{n} x+q_{n}\right)$. Up to a subsequence, we can assume that there exists some $z \in \mathcal{D}^{1,2}\left(\mathbb{R}^{N}\right)$ such that $z_{n} \rightarrow z$ in $\mathcal{D}^{1,2}\left(\mathbb{R}^{N}\right)$ and $z_{n}(x) \rightarrow z(x)$ almost everywhere.

Let $h \in \mathcal{D}\left(\mathbb{R}^{N}\right)$ such that $\operatorname{supp}(h)$ is contained in a ball of radius 1 . The same computation as in (15), the center of the ball being free this time, shows that $z_{n} \rightarrow 0$ in $L_{\text {loc }}^{2^{*}}\left(\mathbb{R}^{N}\right)$. On the other hand it follows from (18) that

$$
\int_{B(1)} K\left(s_{n} R_{n} x+q_{n}\right) z_{n}^{2^{*}} d x=\frac{c}{2}>0,
$$

which leads to a contradiction. Thus, $z \neq 0$. Notice also that

$$
z_{n}(x):=s_{n}{ }^{(N-2) / 2} w_{n}\left(s_{n} x+q_{n}\right)=\left(s_{n} R_{n}\right)^{(N-2) / 2} v_{n}\left(s_{n} R_{n} x+R_{n} q_{n}\right) .
$$

Define $r_{n}=s_{n} R_{n}=o(1)$ and $y_{n}:=R_{n} q_{n}$. We have $r_{n} /\left|y_{n}\right|<2 s_{n}=o(1)$ and, up to a subsequence, $y_{n} \rightarrow y \in \mathbb{R}^{N}$. From LemmaA.4, we infer that $z=K(y)^{(2-N) / 4} U_{0}^{\tau, a}$ 
for some $\tau>0, a \in \mathbb{R}^{N}$, and that

$$
\tilde{v}_{n}(x):=v_{n}(x)-K(y)^{\frac{4}{N-2}} U_{0}^{r_{n} \tau, y_{n}+r_{n} a}(x)
$$

is a Palais-Smale sequence for $J$ at level $d-J(u)-K(y)^{(2-N) / 2} D_{0}$.

In both cases a) and b), from a Palais-Smale sequence $\left(v_{n}\right)$ we have singled out another Palais-Smale sequence $\left(\tilde{v}_{n}\right)$ at a level strictly lower, with a fixed minimum amount of decrease. Arguing recursively, and using Lemma A.1 we infer that this process has to stop after a finite number of steps, and that the last Palais-Smale sequence strongly converges to zero. This gives the result.

4) To conclude the proof, we have to relax the initial assumption stating that $\left(v_{n}\right) \subset H_{0}^{1}(B(R))$ for some fixed $R>0$. In the general case, we claim that there exists $k \in \mathbb{N}_{*}$ such that, up to a subsequence,

$$
f(k):=\liminf _{n \rightarrow+\infty} \int_{B(k+1) \backslash B(k)} K(x) v_{n}{ }^{2^{*}} d x=0 .
$$

Indeed, the previous analysis in $3 \mathrm{~b}$ ) shows that

$$
\text { if } f(k)>0, \text { then } f(k)>\frac{1}{2^{*}} \min _{x} K(x) S^{N / 2} .
$$

Since $\left(v_{n}\right)$ is bounded in $L^{2^{*}}\left(\mathbb{R}^{N}\right)$, it follows that $f(k)=0$ for all but a finite number of $k$. Given such a $k$, we take a cut-off function $\chi \in \mathcal{D}\left(\mathbb{R}^{N}\right)$ satisfying $\chi \equiv 1$ on $B(k)$ and $\chi \equiv 0$ outside $B(k+1)$. We claim that both $\left(\chi v_{n}\right)$ and $\left((1-\chi) v_{n}\right)$ are Palais-Smale sequences for $J$. Indeed, let $h \in \mathcal{D}^{1,2}\left(\mathbb{R}^{N}\right)$; we have

$$
\begin{aligned}
\int_{\mathbb{R}^{N}} \nabla\left(\chi v_{n}\right) \nabla h & =\int_{\mathbb{R}^{N}} \chi \nabla v_{n} \nabla h+\int_{\mathbb{R}^{N}} v_{n} \nabla \chi \nabla h \\
& =\int_{\mathbb{R}^{N}} \nabla v_{n} \nabla(\chi h)-\int_{\mathbb{R}^{N}} h \nabla u \nabla \chi+o(\|h\|) \quad\left(v_{n} \rightarrow 0 \text { in } L_{\mathrm{loc}}^{2}\right) \\
& =\int_{\mathbb{R}^{N}} \lambda \frac{v_{n} \chi h}{|x|^{2}}+\int_{\mathbb{R}^{N}} K v_{n}^{2^{*}} h \chi-\int_{\mathbb{R}^{N}} h \nabla v_{n} \nabla \chi+o(\|h\|),
\end{aligned}
$$

so that

$$
\begin{aligned}
\left\langle J^{\prime}\left(\chi v_{n}\right), h\right\rangle & =\int_{\mathbb{R}^{N}} K v_{n}^{2^{*}}\left(\chi-\chi^{2^{*}}\right) h-\int_{\mathbb{R}^{N}} h \nabla u \nabla \chi+o(\|h\|) \\
& \leq c\left(\int_{B(k+1) \backslash B(k)} v_{n}^{2^{*}}\right. \\
& =o(\|h\|) .
\end{aligned}
$$

This is the required inequality. Clearly, from (19) we also infer that

$$
\int_{\mathbb{R}^{N}} K\left(\chi v_{n}\right)^{2^{*}}+\int_{\mathbb{R}^{N}} K\left((1-\chi) v_{n}\right)^{2^{*}}=\int_{\mathbb{R}^{N}} K\left(v_{n}\right)^{2^{*}}+o(1),
$$

thus the level $d-J(u)$ of $\left(v_{n}\right)$ is integrally split between the two new Palais-Smale sequences $\left(\chi v_{n}\right)$ and $\left((1-\chi) v_{n}\right)$.

Let $\mathcal{K}$ denote the Kelvin transform in $\mathcal{D}^{1,2}\left(\mathbb{R}^{N}\right), \mathcal{K}(u)(x):=|x|^{\frac{2-N}{2}} u\left(x /|x|^{2}\right)$. It is easily seen that the functional $J \circ \mathcal{K}$ has the same expression as $J$ except that $K(x)$ has to be replaced by $K\left(x /|x|^{2}\right)$. Hence, the previous analysis in 3) can be applied to $\mathcal{K}\left((1-\chi) v_{n}\right)$, since this sequence is now a Palais-Smale sequence for $J \circ \mathcal{K}$ in $H_{0}^{1}(B(1 / k))$. From the characterization of $\left(\mathcal{K}\left((1-\chi) v_{n}\right)\right)$ we deduce that of 


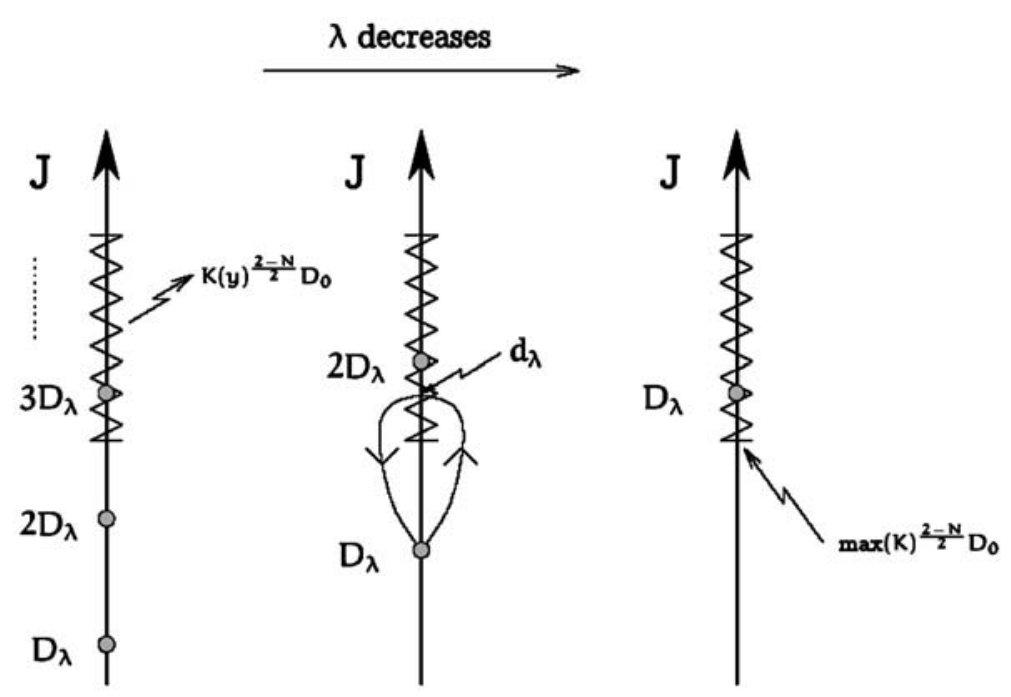

Figure 1.

$\left((1-\chi) v_{n}\right)$; the only point which needs precision concerns $\mathcal{K}\left(U_{0}\left(\frac{x-y_{n}^{j}}{r_{n}^{j}}\right)\right)$, since there is no easy commuting relation between the translations and the Kelvin transform. This is the object of Lemma A.5. The proof is completed.

\section{Existence Results, the case with Palais-Smale}

Once the critical energy regions are identified, one usually tries to construct a minimax scheme which produces a Palais-Smale sequence whose level does not belong to those regions. Of course the easiest part of the energy range to understand is the lowest one. Decreasing the value of $\lambda$ from $(N-2)^{2} / 4$ to 0 , we will make the distinction between three successive cases:

a) $2 D_{\lambda} \leq \max (K)^{\frac{2-N}{2}} D_{0}$,

b) $D_{\lambda}<\max (K)^{\frac{2-N}{2}} D_{0}<2 D_{\lambda}$,

c) $D_{\lambda} \geq \max (K)^{\frac{2-N}{2}} D_{0}$.

Since $D_{\lambda}$ goes to zero as $\lambda$ increases to $(N-2)^{2} / 4$, clearly the first two cases will always happen. Instead, the third one does not happen if $\max (K)=K(0)$. The general situation is outlined in Figure 1. The circular dots represent the discretized energy levels, i.e. the multiples of $D_{\lambda}$. The continuous region represents the energy of a usual single point blow-up, weighted by an appropriate constant depending on the blow-up location. The meaning of $d_{\lambda}$ will be made clear in subsection B) below.

The energy values corresponding to sums of $D_{\lambda}$ and $D_{0}$ terms have not been depicted, since the minimax values that will be constructed will always lie below the smallest of them.

Depending on the behavior of $K$, the solutions will be found either as constrained minimizers (index 1 solutions), or constrained mountain pass points (thought to be index 2 solutions).

A) Constrained minimizers. Remember that the Nehari manifold is denoted by $\mathcal{N}$. We first have the following easy consequence of Theorem 3.1 
Proposition 4.1. Assume that $(\mathcal{H})$ holds. If

$$
\inf _{u \in \mathcal{N}} J(u)<\min \left(D_{\lambda}, \max (K)^{\frac{2-N}{2}} D_{0}\right),
$$

then the infimum is achieved.

Proof. By the Ekeland variational principle, the functional $J$ restricted to $\mathcal{N}$ has a Palais-Smale sequence $\left(u_{n}\right)$ at the infimum level. By definition of $\mathcal{N}$, this sequence is also a Palais-Smale sequence for $J$. By Theorem $3.1\left(u_{n}\right)$ contains a converging subsequence to some minimizer.

Corollary 4.2. Assume that $(\mathcal{H})$ holds, that we are in cases a) or b) and that one of the following conditions holds:

1. $K(x) \geq K(0)$ for all $x \in \mathbb{R}^{N}$.

2. $\exists \varepsilon>0$ such that $\int_{\mathbb{R}^{N}}[K(x)-K(0)]\left(U_{\lambda}^{\varepsilon}\right)^{2^{*}} d x>0$.

Then (1) has at least one positive solution.

Proof. The case $K \equiv K(0)$ is trivial. In the other cases, taking $U_{\lambda}^{\varepsilon}$ as a test function leads to $\tilde{J}\left(U_{\lambda}^{\varepsilon}\right)<D_{\lambda}$. Since we are in one of the cases a) or b), $J$ restricted to $\mathcal{N}$ has a positive minimizer, by Propositions 2.5 and 4.1 .

Corollary 4.3. Assume that $(\mathcal{H})$ holds, that we are in case c) and that $N \leq 4$. Then there exists at least one positive solution of (1).

Proof. This case is very reminiscent of the one studied by Brezis and Nirenberg [11. Let $0 \neq y \in \mathbb{R}^{N}$ such that $K(y)=\max (K)$. It suffices to show that for small epsilons, $\tilde{J}\left(U_{0}^{\varepsilon, y}\right)<K(y)^{(2-N) / 2} D_{0}$. Since $K$ is twice differentiable, it follows from Estimate A.10 that

$$
\int_{\mathbb{R}^{N}} K(x) U_{0}^{\varepsilon, y} d x=\int_{\mathbb{R}^{N}} K(y) U_{0}^{\varepsilon, y} d x+O\left(\varepsilon^{2}\right) .
$$

On the other hand, it follows from Estimate A.6 that

$$
\int_{\mathbb{R}^{N}} \frac{\left(U_{0}^{\varepsilon, y}\right)^{2}}{|x|^{2}} d x= \begin{cases}O_{+}\left(\varepsilon^{2}|\log (\varepsilon)|\right) & \text { if } N=4, \\ O_{+}(\varepsilon) & \text { if } N=3 .\end{cases}
$$

Combining these two estimates in the expression of $\tilde{J}$, we obtain

$$
\left.\tilde{J}\left(U_{0}^{\varepsilon, y}\right) \leq K(y)^{\frac{2-N}{2}} D_{0}-C \varepsilon^{2}|\log (\varepsilon)|\right)
$$

as $\varepsilon \rightarrow 0$, which ends the proof.

Remark 4.4. i) When $N \geq 5$, the previous corollary still holds under the additional assumption that the second derivative of $K$ at one of its maximum points is zero.

ii) When in cases a) or b) one has $K(x) \leq K(0)$ everywhere but $K$ nonconstant, the infimum is never achieved and a higher-dimensional minimax is needed. This is the object of the next subsection.

B) Higher index solutions. In this subsection, we assume that $\inf _{u \in \mathcal{N}} J(u) \geq$ $D_{\lambda}$, the reversed inequality already being treated. Of course, equality holds in this case.

Define

$$
\begin{aligned}
\Gamma:=\left\{\gamma \in \mathcal{C}\left(\mathbb{R}_{*}^{+}, \mathcal{N}\right) \text { s.t. } \exists \varepsilon_{0}>0 \mid \gamma(t)=Q\left(U_{\lambda}^{t}\right) \quad \forall t<\varepsilon_{0}\right. \\
\text { and } \left.\gamma(t)=Q\left(U_{\lambda}^{t}\right) \forall t>\varepsilon_{0}^{-1}\right\},
\end{aligned}
$$


where $Q$ is the central projection on $\mathcal{N}$, and

$$
d_{\lambda}:=\inf _{\gamma \in \Gamma} \max _{t \in \mathbb{R}_{*}^{+}} J(\gamma(t)) .
$$

Notice that since $\inf _{u \in \mathcal{N}} J(u) \geq D_{\lambda}$, the maximum of $J$ along each of these paths is achieved somewhere and thus well defined.

The following proposition holds thanks to an "interaction phenomenon" much in the spirit of the "Bahri and Li" solution [6]. Remember that

$$
a:=\sqrt{1-4 \lambda /(N-2)^{2}}<1 .
$$

Proposition 4.5. Assume that $(\mathcal{H})$ holds and that $a(N-2)<2$; then $d_{\lambda}<2 D_{\lambda}$.

Proof. Let $1>\varepsilon>0$ fixed. We define the path $\gamma \in \Gamma$ such that $\gamma(s):=Q\left(U_{\lambda}^{s}\right)$ if $s<\varepsilon$ or if $s>\varepsilon^{-1}$, and such that $\gamma$ restricted to $\left[\varepsilon, \varepsilon^{-1}\right]$ parameterizes the image by $Q$ of the segment joining $U_{\lambda}^{\varepsilon}$ and $U_{\lambda}^{\frac{1}{\varepsilon}}$. Clearly, if $\varepsilon$ is small enough,

$$
\max _{s \in(0, \varepsilon] \cup\left[\varepsilon^{-1},+\infty\right)} J(\gamma(s))<2 D_{\lambda} .
$$

It suffices then to prove that for $\varepsilon$ small enough,

$$
\max _{t \in[0,1]} \tilde{J}\left((1-t) U_{\lambda}^{\varepsilon}+t U_{\lambda}^{\frac{1}{\varepsilon}}\right)<2 D_{\lambda} .
$$

When $\varepsilon \rightarrow 0$, all the interaction terms disappear so that

$$
\tilde{J}\left((1-t) U_{\lambda}^{\varepsilon}+t U_{\lambda}^{\frac{1}{\varepsilon}}\right)=\frac{\left[t^{2}+(1-t)^{2}\right]^{N / 2}}{\left[t^{2^{*}}+(1-t)^{2^{*}}\right]^{(N-2) / 2}} \cdot D_{\lambda}+o(1) .
$$

The fraction in the previous expression is always strictly less than 2, except if $t=1 / 2$ in which case we need a more precise computation. Let $u:=\frac{1}{2} U_{\lambda}^{\varepsilon}+\frac{1}{2} U_{\lambda}^{\frac{1}{\varepsilon}}$. We have

$$
\int_{\mathbb{R}^{N}}\left[|\nabla u|^{2}-\lambda \frac{u}{|x|^{2}}\right] d x=\frac{1}{2}\left(N D_{\lambda}+\int_{\mathbb{R}^{N}}\left(U_{\lambda}^{\varepsilon}\right)^{2^{*}-1} U_{\lambda}^{\frac{1}{\varepsilon}} d x\right)
$$

and

$$
\int_{\mathbb{R}^{N}} K(x)|u|^{2^{*}} d x=\int_{\mathbb{R}^{N}}|u|^{2^{*}} d x+\int_{\mathbb{R}^{N}}[K(x)-K(0)]|u|^{2^{*}} d x .
$$

Because of the inequality $(x+y)^{2^{*}} \geq x^{2^{*}}+y^{2^{*}}+2^{*} x^{2^{*}-1} y+2^{*} x y^{2^{*}-1}$, valid for nonnegative $x$ and $y$, and because of the equality

$$
\int_{\mathbb{R}^{N}}\left(U_{\lambda}^{\varepsilon}\right)^{2^{*}-1} U_{\lambda}^{\frac{1}{\varepsilon}} d x=\int_{\mathbb{R}^{N}} U_{\lambda}^{\varepsilon}\left(U_{\lambda}^{\frac{1}{\varepsilon}}\right)^{2^{*}-1} d x
$$

obtained by a Kelvin transform, it holds that

$$
\int_{\mathbb{R}^{N}}|u|^{2^{*}} d x \geq\left(\frac{1}{2}\right)^{2^{*}-1}\left(N D_{\lambda}+2^{*} \int_{\mathbb{R}^{N}}\left(U_{\lambda}^{\varepsilon}\right)^{2^{*}-1} U_{\lambda}^{\frac{1}{\varepsilon}} d x\right) .
$$

It follows from Estimate A.13 that

$$
m(\varepsilon):=\int_{\mathbb{R}^{N}}\left(U_{\lambda}^{\varepsilon}\right)^{2^{*}-1} U_{\lambda}^{\frac{1}{\varepsilon}} d x=O_{+}\left(\varepsilon^{a(N-2)}\right),
$$

and from a slight modification of Estimate A.14 that

$$
\int_{\mathbb{R}^{N}}[K(x)-K(0)]|u|^{2^{*}} d x=O\left(\varepsilon^{2}+\varepsilon^{a N}\right)=o\left(\varepsilon^{a(N-2)}\right) .
$$


Hence, as $\varepsilon \rightarrow 0$ we have

$$
\begin{aligned}
\tilde{J}(u) & =\left(\frac{1}{2}-\frac{1}{2^{*}}\right)\left(\int_{\mathbb{R}^{N}}\left[|\nabla u|^{2}-\frac{u^{2}}{|x|^{2}}\right] d x\right)^{N / 2}\left(\int_{\mathbb{R}^{N}} K(x)|u|^{2^{*}} d x\right)^{(2-N) / 2} \\
& \leq \frac{2}{N}\left(N D_{\lambda}+m(\varepsilon)\right)^{N / 2}\left(N D_{\lambda}+2^{*} m(\varepsilon)+o\left(\varepsilon^{a(N-2)}\right)\right)^{(2-N) / 2} \\
& =2 D_{\lambda}\left(1+\left(\frac{D_{\lambda}^{-1}}{2}+o(1)\right) m(\varepsilon)\right)\left(1-\left(D_{\lambda}^{-1}+o(1)\right) m(\varepsilon)\right) \\
& =2 D_{\lambda}-(1+o(1)) O_{+}\left(\varepsilon^{a(N-2)}\right) .
\end{aligned}
$$

The proof is completed.

Remark 4.6. If $K \circ \sigma \in \mathcal{C}^{k}\left(S^{N}\right)$, where $k$ is the first even order for which $D^{k}(K \circ \sigma) \neq$ 0 at the south or north pole, then the condition in the previous proposition can be weakened to $a(N-2)<k$. This follows easily from the proof of Estimate A.14

We now state some easy corollaries of Proposition 4.5

Corollary 4.7. Assume that $(\mathcal{H})$ holds, $N \leq 4$ and we are in case b). Then (1) has at least one positive solution.

Proof. Since $N \leq 4, a(N-2)<2$ for whatever $0<\lambda<(N-2)^{2} / 4$. If $d_{\lambda}=D_{\lambda}$, there exists a sequence $\left(u_{n}\right) \subset \mathcal{D}_{+}^{1,2}\left(\mathbb{R}^{N}\right)$ such that $J\left(u_{n}\right) \rightarrow D_{\lambda}$ and

$$
G\left(u_{n}\right):=\int_{B(0,1)}\left|\nabla u_{n}\right|^{2} d x \cdot\left(\int_{\mathbb{R}^{N}}\left|\nabla U_{\lambda}\right|^{2} d x\right)^{-1}=\frac{1}{2} .
$$

Indeed, for each path $\gamma \in \Gamma, \lim _{s \rightarrow 0} G(\gamma(s))=1$ and $\lim _{s \rightarrow+\infty} G(\gamma(s))=0$. By Ekeland's variational principle, there exists a Palais-Smale sequence $\left(v_{n}\right) \subset \mathcal{D}_{+}^{1,2}\left(\mathbb{R}^{N}\right)$ such that $J\left(v_{n}\right) \rightarrow D_{\lambda}$ and $G\left(v_{n}\right) \rightarrow 1 / 2$. By Theorem 3.1, $\left(v_{n}\right)$ has a subsequence weakly converging to a nonzero solution of (1).

If $d_{\lambda}>D_{\lambda}$, by the Mountain Pass lemma and Proposition 2.5, there is a PalaisSmale sequence for $J$ at level $d_{\lambda}$. By the previous proposition, $d_{\lambda}<2 D_{\lambda}$, and since case a) holds, $d_{\lambda}$ is in the safe energy range.

Corollary 4.8. Assume that $(\mathcal{H})$ holds, $N \leq 4$ and $K$ is radial. Then (I) has at least one positive solution.

Proof. In this case, we can restrict the analysis to the space of radial functions and then use the symmetric criticality principle of Palais (see e.g. [28]). It is clear that blow-up of the classical type is excluded for radial functions, so that all situations reduce to that of case a).

Corollary 4.9. Assume that $(\mathcal{H})$ holds, that $K=1+\varphi$ where $\varphi \in \mathcal{D}\left(\mathbb{R}^{N} \backslash\{0\}\right)$, and that either we are in case a) or $\varphi$ is radial. Then (1) has at least one positive solution.

Proof. This follows from the last remark. 


\section{Existence Results, the CASe without Palais-Smale}

In this section, we analyze the ultimate case which has not yet been treated, namely

$$
D_{\lambda}<\max (K)^{(2-N) / 2} D_{0}<2 D_{\lambda} \quad \text { and } \quad D_{\lambda}<d_{\lambda}<2 D_{\lambda} .
$$

Of course, if $d_{\lambda}<\max (K)^{(2-N) / 2} D_{0}$, the same conclusion as in the previous section holds, the interesting situation is thus the reversed inequality. As already mentioned, the complete analysis is available only in dimension 4 , but some of the intermediate results will be stated in the general setting when available, in view of future use.

Let $y_{0} \in \mathbb{R}^{N}$ such that $d_{\lambda}=K\left(y_{0}\right)^{(2-N) / 2} D_{0}$ and $K^{*}:=\left\{x \in \mathbb{R}^{N}\right.$ s.t. $K(x)=$ $\left.K\left(y_{0}\right)\right\}$. We first assume that $0 \notin K^{*}$, the argument being slightly different. We will indicate at the end of the section how to adapt the proofs in the other case.

It is well known (see e.g. 3]) that for all $\mu>0$, there exists $\nu>0$ such that if $u \in \mathcal{D}^{1,2}\left(\mathbb{R}^{N}\right)$ satisfies $\left\|u-c U_{0}^{\varepsilon, y}\right\|<\nu$ for some $0<\varepsilon<\nu, c>\mu$ and $y \in \mathbb{R}^{N}$, then there exist unique $\varepsilon(u), c(u), y(u), \delta(u)>0$ and $v(u) \in \mathcal{D}^{1,2}\left(\mathbb{R}^{N}\right)$ such that

$$
u=c(u) U_{0}^{\varepsilon(u), y(u)}+\delta(u) v(u)
$$

where $v$ satisfies the orthogonality condition

$$
\left\{\begin{array}{l}
\left\langle U_{0}^{\varepsilon(u), y(u)}, v\right\rangle=0 \\
\left\langle\partial_{y_{i}} U_{0}^{\varepsilon(u), y(u)}, v\right\rangle=\left\langle\partial_{\varepsilon} U_{0}^{\varepsilon(u), y(u)}, v\right\rangle=0, \quad 1 \leq i \leq N,
\end{array}\right.
$$

and $\|v\|=1$. We call $\mathcal{U}$ this set of function. The functions $c(),. \varepsilon(),. y(),. \delta($.$) and$ $v($.$) are smooth in \mathcal{U}$. From now on, we will forget to write down the dependence in $u$ of the coefficients when this will not lead to a confusion.

Given $\rho>0$, we define the $\rho$-neighborhood of the critical set at infinity as

$\mathcal{I}_{\rho}:=\left\{c U_{0}^{\varepsilon, y}+\delta v\right.$ s.t. $\left|c-K\left(y_{0}\right)^{\frac{2-N}{4}}\right|<\rho, \varepsilon<\rho, \delta<\rho, \quad$ and $\left.\operatorname{dist}\left(y, K^{*}\right)<\rho\right\}$, where $\|v\|=1$ and $v$ satisfies $(*)$.

Our aim is to prove that there exist $\rho>0$ and a sequence $\left(\gamma_{n}\right) \subset \Gamma$ such that

$$
\max _{t \in \mathbb{R}_{*}^{+}} J\left(\gamma_{n}(t)\right) \rightarrow d_{\lambda} \quad \text { and } \quad \gamma_{n}\left(\mathbb{R}_{*}^{+}\right) \cap \mathcal{I}_{\rho}=\emptyset \quad \forall n \in \mathbb{N}
$$

Using the Mountain Pass lemma and Theorem 3.1, it will then be easy to conclude that (11) has a positive solution.

The method of proof is inspired by [8]: given a path $\gamma \in \Gamma$ and $t_{0}, t_{1}$ two consecutive intersection times of $\gamma$ with $\mathcal{I}_{\rho}$, we will construct a deformation $\tilde{\gamma}$ of $\gamma$ inside $\left[t_{0}, t_{1}\right]$ which no longer enters into $\mathcal{I}_{\rho}$ without increasing the energy level. To do this, the main step is to connect respectively $\gamma\left(t_{0}\right)$ and $\gamma\left(t_{1}\right)$ to functions for which $\delta=0$ but $\varepsilon>\rho$, without increasing the energy level. The path is then closed moving along the finite-dimensional manifold $\{\delta=0\}$.

Since we will mainly work with $\tilde{J}$, the multiplicative factor $c$ can be brought back to 1 for the crucial estimates.

Let $K_{*}$ be a compact neighborhood of $K^{*}$ contained in $\mathbb{R}^{N} \backslash\{0\}$. The first lemma will give a control on the energy of $\tilde{\gamma}$ on $\{\delta=0\}$.

Lemma 5.1. Let $N=4$. There exists $0<\varepsilon_{1}^{+}<\nu$ such that for all $\varepsilon<\varepsilon_{1}^{+}$there is $\tau>0$ satisfying

$$
\operatorname{dist}\left(y, K^{*}\right)<\tau \Longrightarrow \tilde{J}\left(U_{0}^{\varepsilon, u}\right)<d_{\lambda}
$$


Proof. By Estimates A.10 and A.6. $\exists \varepsilon_{1}^{+}>0$ and $\exists C>0$ such that

$$
\tilde{J}\left(U_{0}^{\varepsilon, y}\right)<K(y)^{(2-N) / 2} D_{0}-C \varepsilon^{2} \log (\varepsilon)
$$

for all $y \in K_{*}$ and $\varepsilon \leq \varepsilon_{1}^{+}$. It suffices then to choose $\tau$ sufficiently small and to use the continuity of $K$.

Lemma 5.2. Let $N=4$. There exists $C^{+}>0,0<\varepsilon_{2}^{+}<\varepsilon_{1}^{+}$and $0<\delta_{2}^{+}<\nu$ such that

$$
\left(\delta \geq C^{+} \varepsilon\right) \Longrightarrow \partial_{\delta} \tilde{J}\left(U_{0}^{\varepsilon, y}+\delta v\right)>0
$$

for all $\varepsilon<\varepsilon_{2}^{+}, \delta<\delta_{2}^{+}, y \in K_{*}$ and $v$ satisfying condition $(*)$ with $\|v\|=1$.

Proof. Remember that

$$
\tilde{J}(u):=\frac{1}{N}\left(\int_{\mathbb{R}^{N}}\left[|\nabla u|^{2}-\lambda \frac{u^{2}}{|x|^{2}}\right] d x\right)^{N / 2}\left(\int_{\mathbb{R}^{N}} K(x)|u|^{2^{*}} d x\right)^{(2-N) / 2} .
$$

Let $u:=U_{0}^{\varepsilon, y}+\delta v$. Because of Estimates A.6 and $\$$.10 we have

$$
\begin{aligned}
\partial_{\delta} \tilde{J}(u)= & \frac{1}{2}\left(N D_{0}+o(1)\right)^{(N-2) / 2}\left(K(y) N D_{0}+o(1)\right)^{(2-N) / 2} \partial_{\delta} A \\
& -\frac{1}{2^{*}}\left(N D_{0}+o(1)\right)^{N / 2}\left(K(y) N D_{0}+o(1)\right)^{-N / 2} \partial_{\delta} B,
\end{aligned}
$$

when $(\varepsilon, \delta) \rightarrow 0$, where $A$ and $B$ are respectively the first and second terms between parentheses in the expression of $\tilde{J}$.

By Estimate A.7

$$
\partial_{\delta} A=2 \delta\left(1-\lambda \int_{\mathbb{R}^{4}} \frac{v^{2}}{|x|^{2}} d x\right)+O(\varepsilon),
$$

and by Estimate A.11,

$$
\partial_{\delta} B=12 \delta K(y) \int_{\mathbb{R}^{4}}\left(U_{0}^{\varepsilon, y}\right)^{2} v^{2} d x+O(\varepsilon)+o(\delta) .
$$

Combining (27) and (28) with (26) yields

$$
\partial_{\delta} \tilde{J}(u)=K(y)^{(2-N) / 2} \delta\left(1-\lambda \int_{\mathbb{R}^{4}} \frac{v^{2}}{|x|^{2}} d x-3 \int_{\mathbb{R}^{4}}\left(U_{0}^{\varepsilon, y}\right)^{2} v^{2} d x\right)+O(\varepsilon)+o(\delta) .
$$

It suffices then to use Proposition 5.3

Proposition 5.3. There exists $c>0$ and $n_{0}>0$ such that $\forall v \in \mathcal{D}^{1,2}\left(\mathbb{R}^{N}\right)$ satisfying the orthogonality condition $(*),\|v\|=1$,

$$
\left(\frac{|y|}{\varepsilon}>n_{0}\right) \Longrightarrow\left(1-\lambda \int_{\mathbb{R}^{N}} \frac{v^{2}}{|x|^{2}} d x-\left(2^{*}-1\right) \int_{\mathbb{R}^{N}}\left(U_{0}^{\varepsilon, y}\right)^{2^{*}-2} v^{2} d x\right)>c .
$$

We divide the proof in different sub-lemmas.

It is well known (see e.g. [7]) that the problem

$$
\left\{\begin{aligned}
-\Delta u & =\sigma U_{0}^{2^{*}-2} u \\
u & \in \mathcal{D}^{1,2}\left(\mathbb{R}^{N}\right)
\end{aligned}\right.
$$

has 1 and $\left(2^{*}-1\right)$ as two first eigenvalues, with eigenspaces being spanned respectively by $\left\langle U_{0}\right\rangle$ and $\left\langle\partial_{y_{i}} U_{0}(i=1, \cdots, N), \partial_{\varepsilon} U_{0}\right\rangle$. We call $\sigma_{3}, \cdots, \sigma_{n}, \cdots$ the successive eigenvalues. 
Define

$$
\alpha:=\frac{1}{2} \min \left[\left(1-\frac{4 \lambda}{(N-2)^{2}}\right),\left(1-\frac{2^{*}-1}{\sigma_{3}}\right)\right] .
$$

Lemma 5.4. There exists $R_{0}>0$ such that $\forall v \in \mathcal{D}^{1,2}\left(\mathbb{R}^{N}\right)$ satisfying $(*),\|v\|=1$,

$$
\left(2^{*}-1\right) \int_{\mathbb{R}^{N}} U_{0}^{2^{*}-2} v^{2} d x<(1-\alpha) \int_{B\left(0, R_{0}\right)}|\nabla v|^{2} d x+\frac{\alpha}{3} .
$$

Proof. Let $p \in \mathbb{N}$ be the index of the first eigenvalue such that $\sigma_{p}>6\left(2^{*}-1\right) / \alpha$, and let $E$ be the direct sum of the eigenspaces corresponding to $\sigma_{3}, \cdots, \sigma_{p}$. Since $E$ is finite dimensional, there exists $R_{0}>$ such that

$$
(u \in E) \Longrightarrow\left\{\begin{array}{l}
\int_{\mathbb{R}^{N}}|\nabla u|^{2} d x \leq \frac{1-\alpha}{1-2 \alpha} \int_{B\left(R_{0}\right)}|\nabla u|^{2} d x \\
2\left(\int_{\mathbb{R}^{N} \backslash B\left(R_{0}\right)}|\nabla u|^{2} d x\right)^{1 / 2}<\alpha / 6 .
\end{array}\right.
$$

Now, if $v \in \mathcal{D}^{1,2}\left(\mathbb{R}^{N}\right),\|v\|=1$, satisfies $(*)$, we decompose $v=v_{E}+w$, where $v_{E} \in E$ and $w \in E^{\perp}$. It yields

$$
\begin{aligned}
\left(2^{*}-1\right) \int_{\mathbb{R}^{N}} U_{0}^{2^{*}-2} v^{2} d x & \leq \frac{2^{*}-1}{\sigma_{3}} \int_{\mathbb{R}^{N}}\left|\nabla v_{E}\right|^{2} d x+\frac{2^{*}-1}{\sigma_{p}} \int_{\mathbb{R}^{N}}|\nabla w|^{2} d x \\
& \leq(1-\alpha) \int_{B\left(R_{0}\right)}\left|\nabla v_{E}\right|^{2} d x+\frac{\alpha}{6} \\
& \leq(1-\alpha) \int_{B\left(R_{0}\right)}|\nabla v|^{2} d x+2\left|\int_{\mathbb{R}^{N} \backslash B\left(R_{0}\right)}\left\langle\nabla v_{E}, \nabla w\right\rangle d x\right|+\frac{\alpha}{6} \\
& \leq \int_{B\left(R_{0}\right)}|\nabla v|^{2} d x+\frac{\alpha}{3},
\end{aligned}
$$

and the proof is complete.

Lemma 5.5. Let $R_{0}>0$. There exists $R_{1}>0$ such that for all $v \in H^{1}\left(B\left(R_{1}\right)\right)$ there exists $\tilde{v} \in H^{1}\left(B\left(R_{1}\right)\right)$ satisfying :

1. $\tilde{v}_{\mid \partial B\left(R_{1}\right)}=v_{\mid \partial B\left(R_{1}\right)}$,

2. $\int_{B\left(R_{1}\right)}|\nabla \tilde{v}|^{2} d x \leq \frac{1-\alpha}{1-2 \alpha} \int_{B\left(R_{1}\right) \backslash B\left(R_{0}\right)}|\nabla v|^{2} d x$.

Proof. Choose $R_{1}$ such that $\frac{R_{1}}{R_{0}}>\left(\frac{1-\alpha}{\alpha}\right)^{2}$, and $s \in\left[R_{0}, R_{1}\right]$ satisfying

$$
\int_{\partial B(s)}\left|\nabla_{t} v\right|^{2}=\min _{r \in\left[R_{0}, R_{1}\right]} \int_{\partial B(r)}\left|\nabla_{t} v\right|^{2},
$$

where $\nabla_{t}$ refers to the tangential gradient (i.e. the gradient of $v$ restricted to $\partial B(r))$. Define $\tilde{v}:=v$ in $B\left(R_{1}\right) \backslash B(s)$ and $\tilde{v}(x):=v(s x /\|x\|)$ in $B(s)$.

By definition of $R_{1}$, either $\frac{s}{R_{0}}>\frac{1-\alpha}{\alpha}$ or $\frac{R_{1}}{s}>\frac{1-\alpha}{\alpha}$. In the first case, we have

$$
\begin{aligned}
\int_{B\left(R_{1}\right)}|\nabla \tilde{v}|^{2} d x & \leq s \int_{\partial B(s)}\left|\nabla_{t} v\right|^{2}+\int_{B\left(R_{1}\right) \backslash B(s)}|\nabla v|^{2} d x \\
& \leq \frac{s}{s-R_{0}} \int_{B(s) \backslash B\left(R_{0}\right)}|\nabla v|^{2} d x+\frac{s}{s-R_{0}} \int_{B\left(R_{1}\right) \backslash B(s)}|\nabla v|^{2} d x \\
& \leq \frac{1-\alpha}{1-2 \alpha} \int_{B\left(R_{1}\right) \backslash B\left(R_{0}\right)}|\nabla v|^{2} d x
\end{aligned}
$$


And in the other case,

$$
\begin{aligned}
\int_{B\left(R_{1}\right)}|\nabla \tilde{v}|^{2} d x & \leq s \int_{\partial B(s)}\left|\nabla_{t} v\right|^{2}+\int_{B\left(R_{1}\right) \backslash B(s)}|\nabla v|^{2} d x \\
& \leq \frac{s}{R_{1}-s} \int_{B\left(R_{1}\right) \backslash B(s)}|\nabla v|^{2} d x+\int_{B\left(R_{1}\right) \backslash B(s)}|\nabla v|^{2} d x \\
& \leq \frac{1-\alpha}{1-2 \alpha} \int_{B\left(R_{1}\right) \backslash B(s)}|\nabla v|^{2} d x .
\end{aligned}
$$

If $s=R_{0}$ or $s=R_{1}$, the terms with $\left(s-R_{0}\right)^{-1}$ or $\left(R_{1}-s\right)^{-1}$ have to be dropped in the inequalities above. The proof is complete.

Lemma 5.6. There exists $n_{0}>0$ such that $\forall v \in \mathcal{D}^{1,2}\left(\mathbb{R}^{N}\right),\|v\|=1, \forall y \in \mathbb{R}^{N}$, $\forall \varepsilon>0$,

$$
\left(\frac{|y|}{\varepsilon}>n_{0}\right) \Longrightarrow \lambda \int_{\mathbb{R}^{N}} \frac{v^{2}}{|x|^{2}} d x<(1-\alpha) \int_{\mathbb{R}^{N} \backslash B\left(y / \varepsilon, R_{0}\right)}|\nabla v|^{2} d x+\frac{\alpha}{3} .
$$

Proof. Let $R_{0}$ and $R_{1}$ given by Lemmas 5.4 and 5.5 We claim that there exists $n_{0}>0$ such that

$$
\left(\frac{|y|}{\varepsilon}>n_{0}\right) \Longrightarrow \lambda \int_{B\left(y / \varepsilon, R_{1}\right)} \frac{v^{2}}{|x|^{2}} d x<\frac{\alpha}{3} .
$$

Indeed, by the Hölder and Sobolev inequalities,

$$
\begin{aligned}
\int_{B\left(y / \varepsilon, R_{1}\right)} \frac{v^{2}}{|x|^{2}} d x & \leq\left(\int_{\mathbb{R}^{N}}|v|^{2^{*}} d x\right)^{2 / 2^{*}} \cdot\left(\int_{B\left(y / \varepsilon, R_{1}\right)}|x|^{-N} d x\right)^{2 / N} \\
& \leq\|v\|^{2} O\left((\varepsilon /|y|)^{2}\right) .
\end{aligned}
$$

Let $\tilde{v} \in \mathcal{D}^{1,2}\left(\mathbb{R}^{N}\right)$ such that $\tilde{v}=v$ outside $B\left(y / \varepsilon, R_{1}\right)$ and $\tilde{v}$ is given by Lemma 5.5 inside $B\left(y / \varepsilon, R_{1}\right)$. We have

$$
\begin{aligned}
\lambda \int_{\mathbb{R}^{N}} \frac{v^{2}}{|x|^{2}} d x & =\int_{B\left(y / \varepsilon, R_{1}\right)} \frac{v^{2}}{|x|^{2}} d x+\lambda \int_{\mathbb{R}^{N} \backslash B\left(y / \varepsilon, R_{1}\right)} \frac{v^{2}}{|x|^{2}} d x \\
& \leq \frac{\alpha}{3}+\frac{4 \lambda}{(N-2)^{2}} \int_{\mathbb{R}^{N}}|\nabla \tilde{v}|^{2} d x \\
& \leq \frac{\alpha}{3}+(1-\alpha) \int_{\mathbb{R}^{N} \backslash B\left(y / \varepsilon, R_{0}\right)}|\nabla v|^{2} d x .
\end{aligned}
$$

Proof of Proposition 5.3. Let $R_{0}, R_{1}$ and $n_{0}$ be given by Lemmas 5.4, 5.5] and 5.6 If $\varepsilon>0$ and $y \in \mathbb{R}^{N}$ are such that $|y| / \varepsilon>n_{0}$,

$$
\left(1-\lambda \int_{\mathbb{R}^{N}} \frac{v^{2}}{|x|^{2}} d x-\left(2^{*}-1\right) \int_{\mathbb{R}^{N}}\left(U_{0}^{\varepsilon, y}\right)^{2^{*}-2} v^{2} d x\right)>\frac{\alpha}{3}>0,
$$

and the proof is complete.

After the dependence in $\delta$, we now analyze the possibility to make deformations in $\varepsilon$. One important difference here is that changing $\varepsilon$ will destroy the orthogonality condition $(*)$. One solution would be to change $v$ accordingly, but this creates other difficulties which we will not discuss here. Instead, we will define a flow in 
a neighborhood of the critical set at infinity which increases $\varepsilon$ while not changing the other parameters too much.

Remember that

$\mathcal{U}:=\left\{u \in \mathcal{D}^{1,2}\left(\mathbb{R}^{N}\right)\right.$ s.t. $\left\|u-c U_{0}^{\varepsilon, y}\right\|<\nu$ for some $0<\varepsilon<\nu, c>\mu$ and $\left.y \in \mathbb{R}^{N}\right\}$.

In $\mathcal{U}$, define the vector field $\Upsilon$

$$
u:=c(u) U_{0}^{\varepsilon(u), y(u)}+\delta(u) v(u) \longmapsto c(u) \partial_{\varepsilon} U_{0}^{\varepsilon, y}{ }_{\mid \varepsilon=\varepsilon(u), y=y(u)},
$$

where v satisfies condition $(*)$ and $\|v\|=1$. By definition of $\mathcal{U}, \Upsilon$ is well defined and locally lipschitz, even if unbounded as $\varepsilon(u) \rightarrow 0$. We call $\eta(., u)$ the flow generated by $\Upsilon$ with initial condition $u$. A priori, $\eta$ is only defined locally.

Lemma 5.7. Let $u_{0} \in \mathcal{U}$. Then $\eta\left(., u_{0}\right)$ is defined as long has $\varepsilon\left(\eta\left(., u_{0}\right)\right)<\nu$, and

- $c\left(\eta\left(., u_{0}\right)\right)=c\left(u_{0}\right)$,

- $\delta\left(\eta\left(., u_{0}\right)\right)=\delta\left(u_{0}\right)$,

- $\left\|y\left(\eta\left(t, u_{0}\right)\right)-y\left(u_{0}\right)\right\| \leq t O\left(\delta\left(u_{0}\right)\right)$,

- $\partial_{t} \varepsilon\left(\eta\left(t, u_{0}\right)\right)=1+O\left(\delta\left(u_{0}\right)\right)$.

Proof. Let $\tilde{\eta}$ be the curve defined by

$$
\tilde{\eta}(t):=c\left(u_{0}\right) U_{0}^{\varepsilon\left(u_{0}\right)+t, y\left(u_{0}\right)}+\delta\left(u_{0}\right) v\left(u_{0}\right),
$$

and $\tilde{c}, \tilde{\varepsilon}, \tilde{y}, \tilde{\delta}, \tilde{v}$ its coordinate functions:

$$
\tilde{\eta}(t)=\tilde{c}(t) U_{0}^{\tilde{\varepsilon}(t), \tilde{y}(t)}+\tilde{\delta}(t) \tilde{v}(t) .
$$

Since $\partial_{t} \tilde{\eta}(0)=\Upsilon\left(u_{0}\right)$, the derivative $\partial_{t} \varepsilon\left(\eta\left(t, u_{0}\right)\right)_{\mid t=0}$ is the same as $\partial_{t} \tilde{\varepsilon}(0)$, and similarly for the other coordinates. Differentiating (33) with respect to $t$ at $t=0$ yields

(34) $\partial_{t} \tilde{c}(0) U_{0}^{\varepsilon, y}+c \partial_{\varepsilon} U_{0}^{\varepsilon, y} \partial_{t} \tilde{\varepsilon}(0)+c\left\langle\nabla_{y} U_{0}^{\varepsilon, y}, \partial_{t} \tilde{y}(0)\right\rangle+\partial_{t} \tilde{\delta}(0) v+\delta \partial_{t} \tilde{v}(0)=c \partial_{\varepsilon} U_{0}^{\varepsilon, y}$.

On the other hand, differentiating the equalities

$$
\|\tilde{v}(t)\|=1 \quad \text { and } \quad\left\langle\tilde{v}(t), U_{0}^{\tilde{\varepsilon}(t), \tilde{y}(t)}\right\rangle=0
$$

at $t=0$, and using the orthogonality property of $v$ yields

$$
\left\langle v, \partial_{t} \tilde{v}(0)\right\rangle=\left\langle\partial_{t} \tilde{v}(0), U_{0}^{\varepsilon, y}\right\rangle=0 .
$$

Taking the scalar product of (34) with $U_{0}^{\varepsilon, y}$ and $v$ respectively gives $\partial_{t} \tilde{c}(0)=$ 0 and $\partial_{t} \tilde{\delta}(0)=0$. The two first claims easily follow. Then, differentiating the orthogonality condition

$$
\left\langle\tilde{v}(t), \partial_{\varepsilon} U_{0}^{\tilde{\varepsilon}(t), \tilde{y}(t)}\right\rangle=\left\langle\tilde{v}(t), \partial_{y_{i}} U_{0}^{\tilde{\varepsilon}(t), \tilde{y}(t)}\right\rangle=0
$$

at $t=0$ and using $\left\|\partial_{\varepsilon}^{2} U_{0}^{\varepsilon, y}\right\|=O\left(\varepsilon^{-2}\right)$, we obtain

$$
\left\langle\partial_{t} \tilde{v}(0), \partial_{\varepsilon} U_{0}^{\varepsilon, y}\right\rangle=O\left(\varepsilon^{-2}\right) \text { and }\left\langle\partial_{t} \tilde{v}(0), \partial_{y_{i}} U_{0}^{\varepsilon, y}\right\rangle=O\left(\varepsilon^{-2}\right) .
$$

Taking the scalar product of (34) with $\partial_{\varepsilon} U_{0}^{\varepsilon, y}$ and $\partial_{y_{i}} U_{0}^{\varepsilon, y}$ respectively, and using the estimate $\left\|\partial_{\varepsilon} U_{0}^{\varepsilon, y}\right\|=0_{+}(\varepsilon)$, yields

$$
\partial_{t} \tilde{\varepsilon}(0)=1+O(\delta) \text { and }\left\|\partial_{t} \tilde{y}(0)\right\|=O(\delta) .
$$

Since by the first step $\tilde{\delta}$ is constant, the third and fourth claims follow. The proof is complete. 
Lemma 5.8. Let $N=4$ and let $C^{+}$be given by Lemma 5.2. There exist $0<\varepsilon_{3}^{+}<$ $\varepsilon_{2}^{+}$and $0<\delta_{3}^{+}<\delta_{2}^{+}$such that

$$
\left(\delta \leq 2 C^{+} \varepsilon\right) \Longrightarrow \partial_{t} \tilde{J}\left(\eta\left(t, U_{0}^{\varepsilon, y}+\delta v\right)\right)_{\mid t=0}<0,
$$

for all $\varepsilon<\varepsilon_{3}^{+}, \delta<\delta_{3}^{+}$and $v \in \mathcal{D}^{1,2}\left(\mathbb{R}^{N}\right)$ satisfying $(*)$ with $\|v\|=1$.

Proof. First observe that

$$
\partial_{t} \tilde{J}\left(\eta\left(t, U_{0}^{\varepsilon, y}+\delta v\right)\right)_{\mid t=0}=\partial_{\varepsilon} \tilde{J}\left(U_{0}^{\varepsilon, y}+\delta v\right)_{\mid \varepsilon=\varepsilon} .
$$

Because of Estimates A.6, A.7 and A.10 we have

$$
\begin{aligned}
\partial_{\varepsilon} \tilde{J}\left(U_{0}^{\varepsilon, y}+\delta v\right)= & \frac{1}{2}\left(N D_{0}+o(1)\right)^{(N-2) / 2}\left(K(y) N D_{0}+o(1)\right)^{(2-N) / 2} \partial_{\varepsilon} A \\
& -\frac{1}{2^{*}}\left(N D_{0}+o(1)\right)^{N / 2}\left(K(y) N D_{0}+o(1)\right)^{-N / 2} \partial_{\varepsilon} B,
\end{aligned}
$$

when $(\varepsilon, \delta) \rightarrow 0$, where $A$ and $B$ were defined in Lemma 5.2 .

Expansion yields

$$
\begin{aligned}
\partial_{\varepsilon} A & =-\partial_{\varepsilon}\left(\int_{\mathbb{R}^{4}} \frac{\left(U_{0}^{\varepsilon, y}\right)^{2}}{|x|^{2}} d x\right)+2\left\langle\partial_{\varepsilon} U_{0}^{\varepsilon, y}, v\right\rangle-2 \delta \int_{\mathbb{R}^{4}} \frac{\partial_{\varepsilon} U_{0}^{\varepsilon, y} v}{|x|^{2}} d x \\
& =-O_{+}(\varepsilon|\log (\varepsilon)|)+O(\delta),
\end{aligned}
$$

by Estimates A.8, A.9 and because of the orthogonality condition (*). For the second term, expanding the fourth power gives

$$
\partial_{\varepsilon} \int_{\mathbb{R}^{4}} K(x)\left(U_{0}^{\varepsilon, y}+\delta v\right)^{4} d x=O(\varepsilon)+O(\delta)+O\left(\delta^{2} \varepsilon^{-1}\right)
$$

by Estimate A.12 Combining (40) and (41) with (39) and using $\delta<2 C^{+} \varepsilon$, we obtain

$$
\partial_{\varepsilon} \tilde{J}\left(U_{0}^{\varepsilon, y}+\delta v\right)=-O_{+}(\varepsilon|\log (\varepsilon)|)+O(\varepsilon)
$$

and the result follows.

We can now prove the key proposition of this section:

Proposition 5.9. Let $N=4$. There exists $\rho>0$ such that for every $\gamma \in \Gamma$ there exists $\tilde{\gamma} \in \Gamma$ satisfying

$$
\tilde{\gamma}\left(\mathbb{R}_{*}^{+}\right) \cap \mathcal{I}_{\rho}=\emptyset \quad \text { and } \quad \max _{t \in \mathbb{R}_{*}^{+}} J(\tilde{\gamma}(t)) \leq \max \left(d_{\lambda}, \max _{t \in \mathbb{R}_{*}^{+}} J(\gamma(t))\right) .
$$

Proof. Let $\varepsilon_{3}^{+}$be given by Lemma 5.8 and let $\tau$ be given by Lemma 5.1 with the choice $\varepsilon=\varepsilon_{3}^{+}$. Without loss of generality, we can assume that $\tau \leq \varepsilon_{3}^{+}$. We claim that $\rho:=\tau / C$ for some sufficiently large $C$ fulfills the thesis. Indeed, let $\gamma \in \Gamma$ and let $\left[t_{0}, t_{1}\right]$ be an interval such that $\gamma\left(\left[t_{0}, t_{1}\right]\right) \subset \mathcal{I}_{\rho}$ and $\gamma\left(t_{0}\right) \cup \gamma\left(t_{1}\right) \subset \partial \mathcal{I}_{\rho}$. We can write $\gamma\left(t_{0}\right) / c\left(\gamma\left(t_{0}\right)\right)$ as $U_{0}^{\varepsilon, y}+\delta v$ for some coefficients satisfying the usual conditions, and similarly in $t_{1}$.

If $\delta>C^{+} \varepsilon$, we know by Lemma 5.2 that we can decrease it until $\delta=C^{+} \varepsilon$, the deformation also decreasing the value of $\tilde{J}$. Once this deformation is performed, we have $\delta<2 C^{+} \varepsilon$ so that by Lemma 5.8 we can follow the flow associated to the vector field $\Upsilon$ and still decrease the value of $\tilde{J}$. This can be done until the corresponding $\varepsilon$ reaches $\varepsilon_{3}^{+}$, but we decide to stop when it reaches $\tau$. If $C$ is large enough, the value of $\tilde{J}$ at this point of the deformation has dropped of a quantity which is $O_{+}\left(\tau^{2}|\log (\tau)|\right)$, as is easily seen from Estimates A.6, A.11 and the expression of 
$\tilde{J}$. Now decreasing $\delta$ to 0 , we will perhaps increase the value of $\tilde{J}$ but we will not reclimb above the initial level $J\left(\gamma\left(t_{0}\right)\right)$. This being done, let us call $U_{0}^{\tau, z_{0}}$ the point that we have reached. We claim that $\tilde{J}\left(U_{0}^{\tau, z_{0}}\right)<d_{\lambda}$. Indeed, because of the choice of $\tau$ and of claims three and four in Lemma 5.7 $z_{0}$ still satisfies the inequality $\operatorname{dist}\left(z_{0}, K^{*}\right)<\tau$; it suffices then to use Lemma 5.1 .

To close the deformation, we perform the same process to $\gamma\left(t_{1}\right) / c\left(\gamma\left(t_{1}\right)\right)$, ending at some $U_{0}^{\tau, z_{1}}$, and then link $U_{0}^{\tau, z_{0}}$ and $U_{0}^{\tau, z_{1}}$ following a path in the set $\{\delta=$ $0\} \cap\left\{\operatorname{dist}\left(y, K^{*}\right)<\tau\right\}$. The deformed path is then lifted back on $\mathcal{N}$ using central projection (which do not affect the value of $\tilde{J}$ ).

Repeating this for each initial intersection of $\gamma$ with $\mathcal{I}_{\rho}$, we construct a path which has the required properties for $\tilde{\gamma}$. The proof is complete.

Remark 5.10. As stated at the start of the section, we have assumed that $0 \notin K^{*}$ in order to avoid including $y$ in the estimates. The proof of the preceding proposition in the other case follows the same series of lemmas, whose proofs have to be slightly adapted. The only main difference is that the estimates in $\varepsilon$ have to be replaced by estimates in $\varepsilon /|y|$ when $y \rightarrow 0$, as obtained in the Appendix, the case $y \rightarrow+\infty$ again being reduced to the case $y \rightarrow 0$ by a Kelvin transform.

Collecting previous partial results, we can now state our main theorem in dimension 4:

Theorem. Assume that $N=4$ and that $K$ and $\lambda$ satisfy assumption $(\mathcal{H})$. Then there exists a positive solution of (1).

\section{A. Appendix}

Lemma A.1. If $\left(u_{n}\right) \subset \mathcal{D}^{1,2}\left(\mathbb{R}^{N}\right)$ is a Palais-Smale sequence for $J$ at level $d$, then $\left(u_{n}\right)$ is bounded and $d \geq 0$.

Proof. Since $\left(u_{n}\right)$ is a Palais-Smale sequence, for large $n$ we have

$$
d+1+\left\|u_{n}\right\| \geq J\left(u_{n}\right)-\frac{1}{2^{*}}\left\langle J^{\prime}\left(u_{n}\right), u_{n}\right\rangle \geq C\left\|u_{n}\right\|^{2} .
$$

Hence $\left(u_{n}\right)$ is bounded in $\mathcal{D}^{1,2}\left(\mathbb{R}^{N}\right)$ and refining the preceding inequality we obtain

$$
d=\lim _{n \rightarrow+\infty} J\left(u_{n}\right)-\frac{1}{2^{*}}\left\langle J^{\prime}\left(u_{n}\right), u_{n}\right\rangle \geq C \limsup _{n \rightarrow+\infty}\left\|u_{n}\right\|^{2} \geq 0 .
$$

Lemma A.2. Let $\left(u_{n}\right) \subset \mathcal{D}^{1,2}\left(\mathbb{R}^{N}\right)$ be a Palais-Smale sequence for $J$ at level $d$. Assume that $u_{n} \rightarrow u$ in $\mathcal{D}^{1,2}\left(\mathbb{R}^{N}\right)$ and $u_{n}(x) \rightarrow u(x)$ almost everywhere. Then $J^{\prime}(u)=0$ and $\left(u_{n}-u\right)$ is a Palais-Smale sequence for $J$ at the level $d-J(u)$.

Proof. The fact that $u$ is a critical point of $J$ follows easily from the weak continuity of $J^{\prime}$. Since $u_{n} \rightarrow u$ in the Hilbert space $\mathcal{D}^{1,2}\left(\mathbb{R}^{N}\right)$, it is clear that

$$
\left\|u_{n}-u\right\|=\left\|u_{n}\right\|-\|u\|+o(1)
$$

and

$$
\int_{\mathbb{R}^{N}} \frac{u_{n}^{2}}{|x|^{2}} d x=\int_{\mathbb{R}^{N}} \frac{\left(u_{n}-u\right)^{2}}{|x|^{2}} d x+\int_{\mathbb{R}^{N}} \frac{u^{2}}{|x|^{2}} d x+o(1) .
$$

According to the Brezis-Lieb lemma 10,

$$
\int_{\mathbb{R}^{N}} K(x)\left|u_{n}\right|^{2^{*}} d x=\int_{\mathbb{R}^{N}} K(x)\left|u_{n}-u\right|^{2^{*}} d x+\int_{\mathbb{R}^{N}} K(x)|u|^{2^{*}} d x+o(1),
$$


so that $J\left(u_{n}-u\right)=d-J(u)+o(1)$. It remains to prove that $J^{\prime}\left(u_{n}-u\right) \rightarrow 0$ in $\mathcal{D}^{1,2}\left(\mathbb{R}^{N}\right)^{\prime}$. The linear part is obvious, hence we just need to prove that

$$
\left|\int_{\mathbb{R}^{N}} K(x)\left(\left|u_{n}\right|^{2^{*}-2} u_{n}-|u|^{2^{*}-2} u-\left|u_{n}-u\right|^{2^{*}-2}\left(u_{n}-u\right)\right) \varphi d x\right|=o(\|\varphi\|) .
$$

The part of the integration restricted to the exterior of a fixed ball of sufficiently large radius $R$ is easily seen to satisfy such a bound (see e.g. [28]). The remaining part is estimated in the literature (see e.g. [12, 28]) under the additional assumption that $u \in L_{\text {loc }}^{\infty}$, a bound which we do not have here. However, it follows from Theorem 2.3 that

$$
u \in \bigcap_{p<p_{\lim }} L_{\mathrm{loc}}^{p}\left(\mathbb{R}^{N}\right), \quad \text { with } \quad p_{\lim }=2^{*} \frac{1}{1-\sqrt{1-4 \lambda /(N-2)^{2}}} .
$$

Choose $2^{*}<p<p_{\text {lim }}$. Since

$$
\begin{aligned}
\left.|| u_{n}\right|^{2^{*}-2} u_{n}-|u|^{2^{*}-2} u-\left|u_{n}-u\right|^{2^{*}-2} & \left(u_{n}-u\right) \mid \\
& \leq C\left(\left|u_{n}-u\right|^{2^{*}-2}|u|+|u|^{2^{*}-2}\left|u_{n}-u\right|\right)
\end{aligned}
$$

we deduce from the Hölder inequality that the left-hand side in (42), restricted to $B(R)$, is bounded by

$$
C\|\varphi\|_{2^{*}}\left(\|u\|_{p}\left\|u_{n}-u\right\|_{q}^{2^{*}-2}+\|u\|_{p}^{2^{*}-2}\left\|u_{n}-u\right\|_{q}\right),
$$

where $1 / 2^{*}+1 / p+\left(2^{*}-2\right) / q=1$. It follows from the choice of $p$ that $q<2^{*}$. Hence, by the Rellich theorem, $\left\|u_{n}-u\right\|_{q} \rightarrow 0$ on $B(R)$ and the result follows.

Lemma A.3. Let $\left(v_{n}\right) \subset \mathcal{D}^{1,2}\left(\mathbb{R}^{N}\right), v_{n} \geq 0$, be a Palais-Smale sequence for $J$ at level $d$. If there exists a sequence $\left(R_{n}\right) \rightarrow 0$ such that $w_{n}(x):=R_{n}^{\frac{N-2}{2}} v_{n}\left(R_{n} x\right)$ converges weakly in $\mathcal{D}^{1,2}\left(\mathbb{R}^{N}\right)$ and almost everywhere to some $w \in \mathcal{D}^{1,2}\left(\mathbb{R}^{N}\right)$, then $w$ solves (II) and the sequence $z_{n}:=v_{n}-R_{n}^{\frac{2-N}{2}} w\left(\frac{x}{R_{n}}\right)$ is a Palais-Smale sequence for $J$ at level $d-D_{\lambda}$.

Proof. By definition and invariance under scaling, we have

$$
\int_{\mathbb{R}^{N}} \nabla w_{n} \nabla \varphi-\frac{\lambda}{|x|^{2}} w_{n} \varphi-K\left(R_{n} x\right)\left|w_{n}\right|^{2^{*}-2} w_{n} \varphi d x=o(\|\varphi\|)
$$

for $\varphi \in \mathcal{D}^{1,2}\left(\mathbb{R}^{N}\right)$. Taking the limit $n \rightarrow+\infty$ we obtain

$$
\int_{\mathbb{R}^{N}} \nabla w \nabla \varphi-\frac{\lambda}{|x|^{2}} w \varphi-|w|^{2^{*}-2} w \varphi d x=0 \quad \forall \varphi \in \mathcal{D}^{1,2}\left(\mathbb{R}^{N}\right),
$$

and $w$ solves (11). According to the Brezis-Lieb lemma, and using the fact that $R_{n} \rightarrow 0$ and $w$ is fixed, we have

$$
\begin{aligned}
\int_{\mathbb{R}^{N}} K\left(R_{n} x\right)\left|w_{n}-w\right|^{2^{*}} d x & =\int_{\mathbb{R}^{N}}\left|K^{1 / 2^{*}}\left(R_{n} x\right) w_{n}-w\right|^{2^{*}} d x+o(1) \\
& =\int_{\mathbb{R}^{N}} K\left(R_{n} x\right)\left|w_{n}\right|^{2^{*}} d x-\int_{\mathbb{R}^{N}}|w|^{2^{*}} d x+o(1) .
\end{aligned}
$$


Hence, again using the scaling invariance,

$$
\begin{aligned}
J\left(z_{n}\right) & =\int_{\mathbb{R}^{N}} \frac{1}{2}\left|\nabla\left(w_{n}-w\right)\right|^{2}-\frac{\lambda}{2} \frac{\left(w_{n}-w\right)^{2}}{|x|^{2}}-\frac{1}{2^{*}} K\left(R_{n} x\right)\left|w_{n}-w\right|^{2^{*}} d x \\
& =\int_{\mathbb{R}^{N}} \frac{1}{2}\left|\nabla w_{n}\right|^{2}-\frac{\lambda}{2} \frac{w_{n}^{2}}{|x|^{2}}-\frac{1}{2^{*}} K\left(R_{n} x\right)\left|w_{n}\right|^{2^{*}} d x-D_{\lambda}+o(1) \\
& =J\left(v_{n}\right)-D_{\lambda}+o(1)=d-D_{\lambda}+o(1) .
\end{aligned}
$$

We now need to show that $J^{\prime}\left(z_{n}\right)=o(1)$. For $\varphi \in \mathcal{D}^{1,2}\left(\mathbb{R}^{N}\right),\left\langle J^{\prime}\left(z_{n}\right), \varphi\right\rangle$ is equal to (46) $\int_{\mathbb{R}^{N}} \nabla\left(w_{n}-w\right) \nabla \varphi_{n}-\lambda \frac{\left(w_{n}-w\right) \varphi_{n}}{|x|^{2}}-K\left(R_{n} x\right)\left|w_{n}-w\right|^{2^{*}-2}\left(w_{n}-w\right) \varphi_{n} d x$, where $\varphi_{n}(x):=R_{n}^{(N-2) / 2} \varphi\left(R_{n} x\right),\left\|\varphi_{n}\right\|=\|\varphi\|$. As in the preceding lemma,

$$
\left|\int_{\mathbb{R}^{N}} K\left(R_{n} x\right)\left(\left|w_{n}\right|^{2^{*}-2} w_{n}-|w|^{2^{*}-2} w-\left|w_{n}-w\right|^{2^{*}-2}\left(w_{n}-w\right)\right) \varphi_{n} d x\right|=o\left(\left\|\varphi_{n}\right\|\right) .
$$

Since $w$ is fixed and $R_{n} \rightarrow 0$, we also obtain

$$
\left.\left|\int_{\mathbb{R}^{N}}\left(K\left(R_{n} x\right)-1\right)\right| w\right|^{2^{*}-2} w \varphi_{n} d x \mid=o\left(\left\|\varphi_{n}\right\|\right) .
$$

Combining the two preceding equalities, we deduce from (46) that

$$
\left\langle J^{\prime}\left(z_{n}\right), \varphi\right\rangle=\left\langle J^{\prime}\left(v_{n}\right), \varphi\right\rangle+o(\|\varphi\|)=o(\|\varphi\|) .
$$

Lemma A.4. Let $\left(v_{n}\right) \subset \mathcal{D}^{1,2}\left(\mathbb{R}^{N}\right), v_{n} \geq 0$, be a Palais-Smale sequence for $J$ at level $d$. Assume that there exist sequences $y_{n} \rightarrow y \in \mathbb{R}^{N}, r_{n} \rightarrow 0 \in \mathbb{R}_{*}^{+}$such that $w_{n}(x):=r_{n}{ }^{\frac{N-2}{2}} v_{n}\left(r_{n} x+y_{n}\right)$ converges weakly in $\mathcal{D}^{1,2}\left(\mathbb{R}^{N}\right)$ and almost everywhere to some $w \in \mathcal{D}^{1,2}\left(\mathbb{R}^{N}\right)$. If $\frac{\left|y_{n}\right|}{r_{n}} \rightarrow+\infty$, then $K(y)^{(N-2) / 4} w$ solves (10) and $z_{n}:=$ $v_{n}-r_{n} \frac{2-N}{2} v_{n}\left(\frac{x-y_{n}}{r_{n}}\right)$ is a Palais-Smale sequence for $J$ at level $d-K(y)^{(2-N) / 2} D_{0}$.

Proof. As in the previous lemma, after scaling we obtain

$$
\int_{\mathbb{R}^{N}} \nabla w_{n} \nabla \varphi-\frac{\lambda}{\left|x-y_{n} r_{n}^{-1}\right|^{2}} w_{n} \varphi-K\left(r_{n} x+y_{n}\right)\left|w_{n}\right|^{2^{*}-2} w_{n} \varphi d x=o(\|\varphi\|),
$$

for $\varphi \in \mathcal{D}^{1,2}\left(\mathbb{R}^{N}\right)$. Since $\left|y_{n}\right| r_{n}^{-1} \rightarrow+\infty$, for each fixed $\varphi$ we have

$$
\lim _{n \rightarrow+\infty} \int_{\mathbb{R}^{N}} \frac{\lambda}{\left|x-y_{n} r_{n}^{-1}\right|^{2}} w_{n} \varphi d x=0 .
$$

Taking the limit as $n \rightarrow+\infty$ in (47), we infer that $-\Delta w-K(y) w^{2^{*}-1}=0$, or equivalently, that $K(y)^{(N-2) / 4} w$ solves (10). We also have

$$
\int_{\mathbb{R}^{N}} \frac{w_{n} w}{\left|x-y_{n} r_{n}^{-1}\right|^{2}} d x=\int_{\mathbb{R}^{N}} \frac{w^{2}}{\left|x-y_{n} r_{n}^{-1}\right|^{2}} d x=o(1),
$$

so that as in (45),

$$
J\left(z_{n}\right)=J\left(v_{n}\right)-K(y)^{(2-N) / 2} D_{0}+o(1)=d-K(y)^{(2-N) / 2} D_{0}+o(1) .
$$

To prove that $J^{\prime}\left(z_{n}\right)=o(1)$, we proceed as in the previous lemma, the only additional estimate to check being

$$
\int_{\mathbb{R}^{N}} \frac{w \varphi_{n}}{\left|x+y_{n} r_{n}^{-1}\right|^{2}} d x=o\left(\left\|\varphi_{n}\right\|\right)
$$


DIDIER SMETS

where $\varphi_{n}(x)=r_{n}^{(N-2) / 2} \varphi\left(r_{n} x+y_{n}\right),\left\|\varphi_{n}\right\|=\|\varphi\|$. This follows from the CauchySchwartz and Hardy inequalities.

Lemma A.5. Let $\mathcal{K}$ denote the Kelvin transform in $\mathbb{R}^{N}$. If $\left(r_{n}\right) \subset \mathbb{R}_{*}^{+}$and $\left(y_{n}\right) \subset$ $\mathbb{R}^{N}$ are sequences such that $\frac{\left|y_{n}\right|}{r_{n}} \rightarrow+\infty$, then in the sense of the $\mathcal{D}^{1,2}\left(\mathbb{R}^{N}\right)$ norm,

$$
r_{n}^{\frac{2-N}{2}} \mathcal{K}\left(U_{0}\left(\frac{x-y_{n}}{r_{n}}\right)\right)=\left(r_{n}\left|y_{n}\right|^{-2}\right)^{\frac{2-N}{2}} U_{0}\left(\frac{x-\frac{y_{n}}{\left|y_{n}\right|^{2}}}{r_{n}\left|y_{n}\right|^{-2}}\right)+o(1) .
$$

Proof. Remember that $U_{0}(x):=c(N)\left(1+|x|^{2}\right)^{(2-N) / 2}$. Since the explicit form of $U_{0}$ is known, the proof is just a verification. The $\mathcal{D}^{1,2}$ norm being invariant under the scaling

$$
v \mapsto \tilde{v}(x):=\left(r_{n}\left|y_{n}\right|^{-2}\right)^{\frac{N-2}{2}} v\left(x r_{n}\left|y_{n}\right|^{-2}+\frac{y_{n}}{\left|y_{n}\right|^{2}}\right),
$$

we can apply it to each side of (48) to check the convergence. The second term becomes $U_{0}+o(1)$, and after a little algebra, the first one is transformed into

$$
U_{0}^{n}(x):=\left(1+r_{n}\left|y_{n}\right|^{-1}\left\langle x, y_{n}\right\rangle+\left(1+r_{n}^{2}\left|y_{n}\right|^{-2}\right)|x|^{2}\right)^{\frac{2-N}{2}} .
$$

Clearly, the condition $r_{n}\left|y_{n}\right|^{-1} \rightarrow 0$ ensures that $U_{0}^{n}$ strongly converges to $U_{0}$ in $\mathcal{D}^{1,2}\left(\mathbb{R}^{N}\right)$. The proof is complete.

Estimate A.6.

$$
\int_{\mathbb{R}^{N}} \frac{\left(U_{0}^{\varepsilon, y}\right)^{2}}{|x|^{2}} d x= \begin{cases}O_{+}(\varepsilon /|y|) & \text { if } N=3 \\ O_{+}\left((\varepsilon /|y|)^{2}|\log (\varepsilon /|y|)|\right) & \text { if } N=4 \\ O_{+}\left((\varepsilon /|y|)^{2}\right) & \text { if } N \geq 5\end{cases}
$$

Proof. Since

$$
\int_{\mathbb{R}^{N}} \frac{\left(U_{0}^{\varepsilon, y}\right)^{2}}{|x|^{2}} d x=\int_{\mathbb{R}^{N}} \frac{\left(U_{0}^{\varepsilon /|y|, y /|y|}\right)^{2}}{|x|^{2}} d x
$$

we can assume without loss of generality that $|y|=1$. We have

$$
\begin{aligned}
\int_{\mathbb{R}^{N}} \frac{\left(U_{0}^{\varepsilon, y}\right)^{2}}{|x|^{2}} d x & =\int_{\mathbb{R}^{N}} \frac{\varepsilon^{N-2}}{|x|^{2}\left(\varepsilon^{2}+|x-y|^{2}\right)^{N-2}} d x \\
& \leq C \varepsilon^{N-2}\left(\int_{\mathbb{R}^{N}} \frac{\left(1+|x-y|^{2}\right)^{2-N}}{|x|^{2}} d x+\int_{\mathbb{R}^{N}} \frac{\left(1+|x|^{2}\right)^{-1}}{\left(\varepsilon^{2}+|x-y|^{2}\right)^{N-2}} d x\right) .
\end{aligned}
$$

The first integral as well as the part of the second one outside $B(y, 1)$ are clearly bounded independently of $\varepsilon$. On $B(y, 1)$, the numerator is bounded by 1 and the remaining is computed explicitly.

Estimate A.7. Let $v \in \mathcal{D}^{1,2}\left(\mathbb{R}^{N}\right)$ satisfying $(*)$ and $\|v\|=1$. Then

$$
\int_{\mathbb{R}^{N}} \frac{U_{0}^{\varepsilon, y} v}{|x|^{2}} d x= \begin{cases}O\left((\varepsilon /|y|)^{1 / 2}\right) & \text { if } N=3, \\ O(\varepsilon /|y|) & \text { if } N=4, \\ O\left((\varepsilon /|y|)^{3 / 2}\right) & \text { if } N=5, \\ O\left((\varepsilon /|y|)^{2}|\log (\varepsilon /|y|)|^{1 / 2}\right) & \text { if } N=6, \\ O\left((\varepsilon /|y|)^{2}\right) & \text { if } N>6 .\end{cases}
$$

Proof. As in the previous estimate, we can assume that $\|y\|=1$. Let $\tilde{v}():.=v(.+y)$; we have

$$
\int_{\mathbb{R}^{N}} \frac{U_{0}^{\varepsilon, y} v}{|x|^{2}} d x=\int_{\mathbb{R}^{N}} \frac{\varepsilon^{(N-2) / 2} \tilde{v}}{|x-y|^{2}\left(\varepsilon^{2}+|x|^{2}\right)^{(N-2) / 2}} d x .
$$


It is easy to see that there exists a constant $C>0$ such that

$$
\frac{1}{|x-y|^{2}\left(\varepsilon^{2}+|x|^{2}\right)^{(N-2) / 2}} \leq C\left[\frac{1}{|x-y|^{2}\left(1+|x|^{2}\right)^{(N-2) / 2}}+\frac{1}{\left(1+|x-y|^{2}\right)\left(\varepsilon^{2}+|x|^{2}\right)^{(N-2) / 2}}\right] .
$$

Using the Cauchy-Schwarz inequality in $L^{2}\left(|x-y|^{-2} d x\right)$ and $L^{2}\left(\left(\varepsilon^{2}+|x|^{2}\right)^{-1} d x\right)$ respectively, we obtain

$$
\begin{aligned}
\int_{\mathbb{R}^{N}} \frac{U_{0}^{\varepsilon, y}|v|}{|x|^{2}} d x & \leq C \varepsilon^{\frac{N-2}{2}}\left(\left(\int_{\mathbb{R}^{N}} \frac{\tilde{v}^{2}}{|x-y|^{2}} d x\right)^{\frac{1}{2}}+\left(\int_{\mathbb{R}^{N}} \frac{\tilde{v}^{2}}{|x|^{2}} d x\right)^{\frac{1}{2}}\left(\int_{\mathbb{R}^{N}} \frac{\left(\varepsilon^{2}+|x|^{2}\right)^{3-N}}{\left(1+|x-y|^{2}\right)^{2}} d x\right)^{\frac{1}{2}}\right) \\
& \leq C \varepsilon^{\frac{N-2}{2}}\left(1+\left(\int_{\mathbb{R}^{N}} \frac{\left(\varepsilon^{2}+|x|^{2}\right)^{3-N}}{\left(1+|x-y|^{2}\right)^{2}}\right)^{\frac{1}{2}} d x\right) .
\end{aligned}
$$

It then suffices to evaluate the last integral. Outside of the unit ball, the integral is clearly uniformly bounded with respect to $\varepsilon$. On the unit ball, the term in $\left(1+|x-y|^{2}\right)^{-2}$ is bounded by 1 and the remaining integral can be explicitly computed.

Remark. Notice that whereas in dimension 3 the previous estimate gives nothing more than Estimate A.6 combined with the Cauchy-Schwartz and Hardy inequalities, from dimension 4 it traduces a splitting phenomenon between the singularity and the concentrating instanton.

Estimate A.8. Let $N=4$. Then

$$
\partial_{\varepsilon} \int_{\mathbb{R}^{4}} \frac{\left(U_{0}^{\varepsilon, y}\right)^{2}}{|x|^{2}} d x=|y|^{-1} O_{+}((\varepsilon /|y|)|\log (\varepsilon /|y|)|), \quad \text { as } \quad \varepsilon /|y| \rightarrow 0 .
$$

Proof. Follow the same lines as the one of Estimate A.6, replacing $\partial_{\varepsilon} U_{0}^{\varepsilon, y}$ by its explicit expression.

Estimate A.9. Let $N=4$ and $v \in \mathcal{D}^{1,2}\left(\mathbb{R}^{4}\right)$ satisfying $(*)$ and $\|v\|=1$. Then

$$
\partial_{\varepsilon} \int_{\mathbb{R}^{4}} \frac{U_{0}^{\varepsilon, y} v}{|x|^{2}} d x=|y|^{-1} O(1), \quad \text { as } \quad \varepsilon /|y| \rightarrow 0 .
$$

Proof. Follow the same lines as the one of Estimate A.7, replacing $\partial_{\varepsilon} U_{0}^{\varepsilon, y}$ by its explicit expression.

Estimate A.10.

$$
\int_{\mathbb{R}^{N}} K(x)\left(U_{0}^{\varepsilon, y}\right)^{2^{*}} d x=K(y) N D_{0}+O\left(\varepsilon^{2}\right) .
$$

Proof. Let $\varphi \in \mathcal{D}(B(y, 1))$ such that $\varphi \equiv 1$ on $B(y, 1 / 2)$ and $\varphi$ is even with respect to $y$. By oddness, we obtain

$$
\begin{aligned}
& \int_{\mathbb{R}^{N}} K(x)\left(U_{0}^{\varepsilon, y}\right)^{2^{*}} d x \\
= & \int_{\mathbb{R}^{N}} K(y)\left(U_{0}^{\varepsilon, y}\right)^{2^{*}} d x+\int_{\mathbb{R}^{N}}[K(x)-K(y)-\langle\nabla K(y) \mid x-y\rangle \varphi]\left(U_{0}^{\varepsilon, y}\right)^{2^{*}} d x \\
= & K(y) N D_{0}+\int_{\mathbb{R}^{N}}[K(x)-K(y)-\langle\nabla K(y) \mid x-y\rangle \varphi]\left(U_{0}^{\varepsilon, y}\right)^{2^{*}} d x .
\end{aligned}
$$

Since $K$ is twice differentiable and bounded, there exists $C>0$ such that

$$
|K(x)-K(y)-\langle\nabla K(y) \mid x-y\rangle \varphi(x)| \leq C \frac{|x-y|^{2}}{1+|x-y|^{2}} .
$$


By a change of variable,

$$
\int_{\mathbb{R}^{N}} \frac{|x-y|^{2}}{1+|x-y|^{2}}\left(U_{0}^{\varepsilon, y}\right)^{2^{*}} d x=\varepsilon^{2} \int_{\mathbb{R}^{N}} \frac{|x|^{2}}{1+\varepsilon^{2}|x|^{2}}\left(U_{0}\right)^{2^{*}} d x=O\left(\varepsilon^{2}\right),
$$

because $\int_{\mathbb{R}^{N}}|x|^{2}\left(U_{0}\right)^{2^{*}} d x<+\infty$. The proof is complete.

Estimate A.11. Let $v \in \mathcal{D}^{1,2}\left(\mathbb{R}^{N}\right)$ satisfying $(*)$ and $\|v\|=1$. Then

$$
\int_{\mathbb{R}^{N}} K(x)\left(U_{0}^{\varepsilon, y}\right)^{2^{*}-1} v d x=O(\varepsilon) .
$$

Proof. Since $U_{0}^{\varepsilon, y}$ solves (10), and since $(*)$ is satisfied,

$$
\begin{aligned}
& \int_{\mathbb{R}^{N}} K(x)\left(U_{0}^{\varepsilon, y}\right)^{2^{*}-1} v d x \\
= & \int_{\mathbb{R}^{N}} K(y)\left(U_{0}^{\varepsilon, y}\right)^{2^{*}-1} v d x+\int_{\mathbb{R}^{N}}[K(x)-K(y)]\left(U_{0}^{\varepsilon, y}\right)^{2^{*}-1} v d x \\
= & K(y) \int_{\mathbb{R}^{N}} \nabla U_{0}^{\varepsilon, y} \nabla v d x+\int_{\mathbb{R}^{N}}[K(x)-K(y)]\left(U_{0}^{\varepsilon, y}\right)^{2^{*}-1} v d x \\
= & \int_{\mathbb{R}^{N}}[K(x)-K(y)]\left(U_{0}^{\varepsilon, y}\right)^{2^{*}-1} v d x .
\end{aligned}
$$

By the differentiability and boundedness of $K$, there exists $C>0$ such that

$$
|K(x)-K(y)| \leq C \frac{|x-y|}{1+|x-y|} \quad \forall x \in \mathbb{R}^{N} .
$$

Hence, after a change of variable,

$$
\begin{aligned}
\int_{\mathbb{R}^{N}}|K(x)-K(y)|\left(U_{0}^{\varepsilon, y}\right)^{2^{*}-1}|v| d x & \leq C \varepsilon\left(\int_{\mathbb{R}^{N}}|x|^{\frac{2 N}{N+2}}\left(U_{0}\right)^{2^{*}} d x\right)^{\frac{N+2}{2 N}}\|v\|_{2^{*}}^{\frac{1}{2^{*}}} \\
& \leq C \varepsilon
\end{aligned}
$$

by the Sobolev inequality and because $\int_{\mathbb{R}^{N}}|x|^{\frac{2 N}{N+2}}\left(U_{0}\right)^{2^{*}} d x<+\infty$.

Estimate A.12. Let $N=4$, let $v \in \mathcal{D}^{1,2}\left(\mathbb{R}^{4}\right)$ satisfy $(*)$ and let $\|v\|=1$. Then

$$
\partial_{\varepsilon} \int_{\mathbb{R}^{4}} K(x)\left(U_{0}^{\varepsilon, y}+\delta v\right)^{4}=O\left(\varepsilon+\delta+\delta^{2} \varepsilon^{-1}\right) \text { as }(\varepsilon, \delta) \rightarrow 0 .
$$

Proof. Expand the fourth power and use the same tricks as in Estimates A.6, A.7 and A.11. The details are left to the reader.

Estimate A.13. Let $a:=\sqrt{1-4 \lambda /(N-2)^{2}}$. Then

$$
\int_{\mathbb{R}^{N}}\left(U_{\lambda}^{\varepsilon}\right)^{2^{*}-1} U_{\lambda}^{\frac{1}{\varepsilon}} d x=O_{+}\left(\varepsilon^{a(N-2)}\right) .
$$

Proof. By definition of $U_{\lambda}$, we have

$$
\int_{\mathbb{R}^{N}}\left(U_{\lambda}^{\varepsilon}\right)^{2^{*}-1} U_{\lambda}^{\frac{1}{\varepsilon}} d x=C \varepsilon^{2 a} \int_{0}^{\infty}\left[r^{1-a}\left(\varepsilon^{2 a}+r^{2 a}\right)\right]^{-\frac{N+2}{2}}\left[r^{1-a}\left(\varepsilon^{-2 a}+r^{2 a}\right)\right]^{\frac{2-N}{2}} r^{N-1} d r .
$$

We decompose the domain of integration in three parts, depending on whether $|x|<\varepsilon, \varepsilon<|x|<\varepsilon^{-1}$ or $|x|>\varepsilon^{-1}$. On the first part we obtain

$$
\int_{|x|<\varepsilon}\left(U_{\lambda}^{\varepsilon}\right)^{2^{*}-1} U_{\lambda}^{\frac{1}{\varepsilon}} d x \sim \varepsilon^{-2 a} \int_{0}^{\varepsilon} r^{(a-1) N} r^{N-1} d r \sim \varepsilon^{a(N-2)},
$$


on the second part

$$
\int_{\varepsilon<|x|<\varepsilon^{-1}}\left(U_{\lambda}^{\varepsilon}\right)^{2^{*}-1} U_{\lambda}^{\frac{1}{\varepsilon}} d x \sim \varepsilon^{a N} \int_{\varepsilon}^{\varepsilon^{-1}} r^{-N-2 a} r^{N-1} d r \sim \varepsilon^{a(N-2)},
$$

and on the last part

$$
\int_{|x|>\varepsilon^{-1}}\left(U_{\lambda}^{\varepsilon}\right)^{2^{*}-1} U_{\lambda}^{\frac{1}{\varepsilon}} d x \sim \varepsilon^{2 a} \int_{\varepsilon^{-1}}^{\infty} r^{-(1+a) N} r^{N-1} d r \sim \varepsilon^{a(N+2)},
$$

where $\alpha \sim \beta$ means that $\alpha / \beta$ is uniformly bounded above and below by two positive constants as $\varepsilon \rightarrow 0$. Summing up we have the required estimate.

Estimate A.14. Let $a:=\sqrt{1-4 \lambda /(N-2)^{2}}$. Then

$$
\int_{\mathbb{R}^{N}}[K(x)-K(0)]\left(U_{\lambda}^{\varepsilon}\right)^{2^{*}} d x=\left\{\begin{array}{l}
O\left(\varepsilon^{2}+\varepsilon^{a N}\right) \quad \text { if } a N \neq 2, \\
O\left(\varepsilon^{2}|\log (\varepsilon)|\right) \quad \text { if } a N=2 .
\end{array}\right.
$$

Proof. By using a Kelvin transform it is enough to consider the integral restricted to the unit ball. Exactly as in Estimate A.10, we obtain

$$
\int_{B(1)}|K(x)-K(0)|\left(U_{\lambda}^{\varepsilon}\right)^{2^{*}} d x \leq C \varepsilon^{2} \int_{B(1 / \varepsilon)}|x|^{2}\left(U_{\lambda}\right)^{2^{*}} d x .
$$

If $a N>2$, the last integral is bounded uniformly with respect to $\varepsilon$ and the result follows. If $a N \leq 2$, we have

$$
\begin{aligned}
\int_{B(1 / \varepsilon)}|x|^{2}\left(U_{\lambda}\right)^{2^{*}} d x & =C \int_{0}^{1 / \varepsilon} r^{N+1-(1-a) N}\left(1+r^{2 a}\right)^{-N} d r \\
& =\left\{\begin{array}{l}
O\left(\varepsilon^{a N-2}\right) \quad \text { if } a N<2 \\
O(|\log (\varepsilon)|) \quad \text { if } a N=2 .
\end{array}\right.
\end{aligned}
$$

\section{ACKNOWLEDGMENTS}

The main part of this work was done while the author was a TMR postdoc at "Universita La Sapienza", Roma in 2001. Many thanks to the members of the department there for their unmatchable hospitality. I also thank A. Ambrosetti and Y.Y. Li for a short but enlightening discussion in Paris, May 2001, as well as E. Hebey for new references.

\section{REFERENCES}

[1] T. Aubin, Equations différentielles non linéaires et problème de Yamabe concernant la courbure scalaire, J. Math. Pures Appl. 55 (1976), 269-296. MR0431287 (55:4288)

[2] T. Aubin, Problèmes isopérimétriques et espaces de Sobolev, C. R. Acad. Sci. Paris Sér. A-B 280 (1975), 279-281. MR0407905 (53:11675)

[3] A. Bahri, Critical points at infinity in some variational problems, Pitman Res. Notes in Math. Ser. 182, Longman Scientific \& Techn., 1989. MR1019828 (91h:58022)

[4] A. Bahri and H. Brezis, Non-linear elliptic equations on Riemannian manifolds with the Sobolev critical exponent, Topics in geometry, 1-100, Progr. Nonlinear Diff. Eqns Appl. 20, Birkhäuser, Boston, 1996. MR1390310 (97c:53056)

[5] A. Bahri and J.-M. Coron, The scalar-curvature problem on standard three dimensional sphere, J. of Funct. Anal. 95 (1991), 106-172. MR1087949 (92k:58055)

[6] A. Bahri and Y.Y. Li, On a min-max procedure for the existence of a positive solution for certain scalar field equations in $R^{N}$, Rev. Mat. Iberoamericana 6 (1990), 1-15. MR1086148 (92b:35054) 
[7] G. Bianchi and H. Egnell, A note on the Sobolev inequality, J. of Funct. Anal. 100 (1991), 18-24. MR1124290 (92i:46033)

[8] G. Bianchi and H. Egnell, A variational approach to the equation $\Delta u+K u^{(N+2) /(N-2)}=0$ in $\mathbb{R}^{N}$, Arch. Rat. Mech. Anal. 122 (1993), 159-182. MR1217589 (94g:35079)

[9] H. Brezis and T. Kato, Remarks on the Schrödinger operator with singular complex potentials, J. Math. Pures et Appl. 58 (1979), 137-151. MR 0539217 (80i:35135)

[10] H. Brezis and E. Lieb, A relation between pointwise convergence of functions and convergence of functionals, Proc. Amer. Math. Soc. 88 (1983), 486-490. MR0699419 (84e:28003)

[11] H. Brezis and L. Nirenberg, Positive solutions of nonlinear elliptic equations involving critical Sobolev exponents, Comm. Pure and Appl. Math. 36 (1983), 437-477. MR0709644 (84h:35059)

[12] G. Cerami, D. Fortunato and M. Struwe, Bifurcation and multiplicity results for nonlinear elliptic problems involving critical Sobolev exponents, Ann. IHP Anal. Non Lin. 1 (1984), 341-350. MF 0779872 (86e:35016)

[13] O. Druet, The best constants problem in Sobolev inequalities, Mathematische Annalen, 314 (1999), 327-346. MR.1697448 (2000d:58033)

[14] O. Druet, Elliptic equations with critical Sobolev exponents in dimension 3, Annales de l'Institut Henri Poincaré, Analyse non linéaire 19 (2002), 125-142. MR1902741 (2003f:35104)

[15] O. Druet, E. Hebey and F. Robert, $A C^{0}$-theory for the blow-up of second order elliptic equations of critical Sobolev growth, Electronic Research Announcements of the AMS, 9 (2003), 19-25. MR1988868 (2004c:58046)

[16] Z.C. Han, Asymptotic approach to singular solutions for nonlinear elliptic equations involving critical Sobolev exponent, Annales de l'Institut Henri Poincaré, Analyse non linéaire 8 (1991), 159-174. MF1096602 (92c:35047)

[17] E. Hebey and M. Vaugon, The best constant problem in the Sobolev embedding theorem for complete Riemannian manifolds, Duke Mathematical Journal 79 (1995), 235-279. MR.1340298 (96c:53057)

[18] J.L. Kazdan and F.W. Warner, Scalar curvature and conformal deformation of Riemannian strructure, Journal of Differential Geometry 10 (1975), 113-134. MR0365409 (51:1661)

[19] Y.Y. Li, Prescribing scalar curvature on $\mathbb{S}^{n}$ and related problems, Part I, J. Diff. Eqns 120 (1995), 319-410. MR1347349 (98b:53031)

[20] Y.Y. Li, Prescribing scalar curvature on $\mathbb{S}^{n}$ and related problems, Part II, Comm. Pure Appl. Math. 49 (1996), 541-597. MR1383201 (98f:53036)

[21] Y.Y. Li and M. Zhu, Yamabe type equations on three dimensional Riemannian manifolds, Comm. Contemp. Math. 1 (1999), 1-50. MR1681811 (2000m:53051)

[22] P.-L. Lions, The concentration-compactness principle in the calculus of variations. The limit case, Rev. Mat. Ibero Americana 1 (1985), 45-121. MR0850686 (87j:49012)

[23] P. Sacks and K. Uhlenbeck, The existence of minimal immersions of the 2-spheres, Ann. Math. 113 (1981), 1-24. MR0604040 (82f:58035)

[24] R. Schoen, Conformal deformation of a Riemannian metric to constant scalar curvature, J. Diff. Geom. 20 (1984), 479-495. MR0788292 (86i:58137)

[25] R. Schoen, Variational theory for the total scalar curvature functional for Riemannian metrics and related topics, Topics in Calculus of Variations, 120-154, Lecture Notes in Mathematics, Springer, 1989. MR0994021 (90g:58023)

[26] M. Struwe, A global compactness result for elliptic boundary value problems involving limiting nonlinearities, Math. Z. 187 (1984), 511-517. MR0760051|(86k:35046)

[27] S. Terracini, On positive solutions to a class of equations with a singular coefficient and critical exponent, Adv. Dif. Eqns 2 (1996), 241-264. MR.1364003 (97b:35057)

[28] M. Willem, Minimax Theorems, Progr. Nonlinear Diff. Eqns Appl. 24, Birkhäuser, Boston, 1996. MR1400007 (97h:58037)

[29] H. Yamabe, On a deformation of Riemannian structures on compact manifolds, Osaka Math. J. 12 (1960), 21-37. MR0125546 (23:A2847)

Laboratoire J.L. Lions, Université Pierre \& Marie Curie, 175 rue du chevaleret, 75013 Paris, France

E-mail address: smets@ann.jussieu.fr 